Eine ideale eindimensionale statistische Verteilung enthält als Fourier-Komponente sämtliche Wellenlängen von 0 bis $\infty$ mit gleicher Amplitude. Ein in sich zurücklaufendes Muster auf einem Zylinder besitzt aber eine Komponente mit der größten Wellenlänge von $\lambda=360^{\circ}$, die nächstkleineren sind $180^{\circ}, 120^{\circ}, 90^{\circ}, 72^{\circ}$ usf., und erst bei noch kleineren Werten tritt eine dichtere Folge von Wellenlängen auf. Nur dort, wo die kleineren Wellenlängen für den Hauptanteil der Reaktion verantwortlich sind, ist daher eine Annäherung an die theoretische Kurve des Idealfalles des bewegten statistischen Helligkeitsmusters zu erwarten. Dies betrifft das Gebiet geringer Geschwindigkeiten, denn wie in Abschnitt 6 a dargelegt ist, liegen die Wirkungsmaxima kurzwelliger Sinuskomponenten bei kleineren Geschwindigkeiten; damit wird der Bereich der negativen Reaktionen zuverlässig wiedergegeben.

Aber auch im Bereich kurzer Wellenlängen weichen die verwendeten Muster Abb. 15 vom idealen statistischen Muster ab; sie besitzen keine unendlich feine, sondern eine recht grobe Struktur, enthalten also von einem Optimalwert an die kleineren Wellenlängen in abnehmendem Maße. Dieser Mangel ist bei dem breitstreifigen Muster 15 a natürlich viel größer als bei dem feiner gegliederten von $15 \mathrm{~b}$; daher kommt es vermutlich, daß durch langsame Geschwindigkeiten von Muster 15 a keine negativen Reaktionen ausgelöst wurden (s. $1958^{1}$, Abb. 4 und vgl. mit Abb. 4 der Arbeit von Reichardt und $\mathrm{V}_{\text {ARJ }}{ }^{4}$ ).
Damit ist die dritte in der Einleitung gestellte Frage in folgendem Sinne beantwortet: Mit einem Streifenzylinder läßt sich die Situation des bewegten idealen statistischen Musters nur unvollkommen verwirklichen. In demjenigen Geschwindigkeitsbereich, der die statistischen Züge des experimentell dargebotenen Musters am deutlichsten in der Reaktion zum Ausdruck kommen läßt, liegt aber zum Glück der interessanteste Teil der Reaktionskurve: Ein Bereich negativer optokinetischer Wendetendenz. Er läßt unmittelbar auf die Wirksamkeit einer übertragenden Instanz bestimmter Charakteristik ${ }^{4}$ schließen, deren Funktionswert auf eine einseitige Auslenkung hin vorübergehend über die Ruhelage hinaus zur Gegenseite überschwingt.

Meiner Frau danke ich für das Sammeln und Versenden der Versuchstiere, Herrn stud. Dietmar Todt für freiwillige wissenschaftliche Mitarbeit, Fräulein Angela Herrmann und Fräulein Ulrike Schönfeld für gewissenhafte und interessierte technische Mitarbeit, Herrn cand. Peter Kunze für kritische Durchsicht des Manuskripts, und Dr. Werner Reichardt und Dr. Dezsö Varjú, ohne deren theoretische Untersuchungen diese Arbeit nicht zustandegekommen wäre, für entscheidende Anregungen und hilfreiche Kritik.

\title{
Übertragungseigenschaften im Auswertesystem für das Bewegungssehen
}

(Folgerungen aus Experimenten an dem Rüsselkäfer Clorophanus viridis)

\author{
Von Werner Reichardt und Dezsö Varjé
}

Aus dem Max-Planck-Institut für Biologie in Tübingen, Forschungsgruppe Kybernetik

(Z. Naturforschg. 14 b, $674-689$ [1959]; eingegangen am 15. Mai 1959)

\begin{abstract}
Optokinetic reactions of the beetle Chlorophanus are not only elicited by moving figures distinguishable against their backgrounds, but also from movements of randomly constructed patterns of shades from white through black (random functions). The evaluation of relative motions by the central nervous system is made in accordance with the correlation principle ${ }^{1}$. The functional structure of perception, discussed in an earlier paper ${ }^{1}$, describes the reactions to motions of random functions but does not explain the strength of optokinetic reactions which are released by moving sinusoidal patterns. In the following paper the frequency response functions of the filters are determined in such a way that the functional structure (Fig. 1) describes the reactions to both sinusoidal and randomly constructed light patterns (Fig. 3 and 4). Two results follow from the analysis: 1 . The time constants of the low pass filters are $\tau_{\mathrm{F}}=1,6 \mathrm{sec}, \tau_{\mathrm{H}}=0,03 \mathrm{sec}$. 2 . The input filters cannot be of the differentiating type. Their frequency response function is proportional to the root of frequency. The kinetics of the low pass filters can be explained on the basis of a first order linear differential equation which corresponds to a monomolecular chemical reaction. The frequency response functions of the input filters can only be explained by a partial differential equation describing processes of diffusion.
\end{abstract}

In einer früheren Arbeit ${ }^{1}$ wurde auf der Basis der Funktionsstruktur für das Bewegungssehen des Rüsselkäfers Chlorophanus ${ }^{2,3}$ vorausgesagt, daß optomotorische Reaktionen nicht nur bei der Bewegung von Figuren, sondern auch bei der Bewegung von solchen Hellig. keitsmustern auftreten müßten, innerhalb deren die vorkommenden Helligkeitswerte statistisch voneinander un- abhängig sind (Helligkeits-Rauschfunktionen). Die Versuche ${ }^{4}$ haben diese Voraussage bestätigt.

1 W. Reichardt, Z. Naturforschg. 12 b, 448 [1957].

2 B. Hassenstein u. W. Reichardt, Z. Naturforschg. 11 b, 513 [1956].

3 B. Hassenstein, Z. vergleich. Physiol. 40, 556 [1958].

4 B. Hassenstein, Z. Naturforschg. 13 b, 1 [1958]. 
Darüber hinaus ermöglichten die experimentellen Ergebnisse eine näherungsweise Bestimmung der Übertragungs-Eigenschaften der beteiligten zentralnervösen Elemente (Filter). Es erschien uns jedoch notwendig, die Übertragungs-Eigenschaften der Filter nicht nur mit Helligkeits-Rauschfunktionen, also einem Gemisch von F o u rier-Komponenten statistisch verteilter Phasen, sondern auch mit einer F o u ri e r - Komponente zu prüfen. Experimentell mußte man hierzu vor dem Ommatidienraster eine sinusförmige Helligkeitsverteilung vorbeibewegen und die auf verschiedene Geschwindigkeiten erfolgenden optomotorischen Reaktionen registrieren ${ }^{5}$. Der Vorteil dieser Messung gegenüber der mit Helligkeits-Rauschfunktionen bestand u. a. darin, daß bei hinreichend groß gewählter Wellenlänge der Helligkeitsverteilung die Apertur der Ommatidien des Facettenauges praktisch keinen störenden Einfluß auf das Reaktionsergebnis besitzen konnte. Das Ergebnis dieser experimentellen Untersuchung zeigte eine quantitative Abweichung von der theoretisch vorausberechneten Reaktionskurve. Es ist daher notwendig zu untersuchen, welche Veränderungen in den Übertragungs-Eigenschaften der Filter vorzunehmen sind, damit die Theorie sowohl den periodischen als auch den statistischen Reizfall befriedigend wiedergeben kann.

$\mathrm{Zu}$ diesem $\mathrm{Zweck}$ gehen wir von einer verallgemeinerten Funktionsstruktur für das Bewegungssehen aus. Sie ist in Abb. 1 dargestellt. Die elementare Funktionsstruktur enthält zwei lichtempfindliche Rezeptoren A, B, die die Sehelemente (Ommatidien) der Facettenaugen repräsentieren. Das raumzeitliche Geschehen in der optischen Umwelt wird in den Rezeptoren in ein zeitliches umgewandelt. Bei einer Verschiebung des Helligkeitsmusters von links nach rechts wird jeder Helligkeitswert zuerst von Element A und danach um das Zeitintervall $A t$ später von Element B aufgenommen. Das Zeitintervall ist mit der Geschwindigkeit $w$ der Musterbewegung durch die Beziehung $\Delta t=\Delta s / w$ verknüpft, wenn man mit $\Delta s$ die Entfernung zwischen $\mathrm{A}$ und $\mathrm{B}$ bezeichnet. Die zeitlich veränderlichen Eingangsgrößen von $A$ und $B$ kennzeichnen wir mit $L_{\mathrm{A}}, L_{\mathrm{B}}$. Sie stellen - abgesehen von einem möglichen Proportionalitätsfaktor - Zeitfunktionen dar, die von den Filtern $\mathrm{D}_{\mathrm{A}}, \mathrm{D}_{\mathrm{B}}$ in die zeitabhängigen Vorgänge $L^{*}{ }_{\mathrm{A}}, L^{*}{ }_{\mathrm{B}}$ transformiert werden. Nach weiterer Transformation von $L^{*}{ }_{\mathrm{A}}$ in den Filtern $\mathrm{F}_{\mathrm{A}}, \mathrm{H}_{\mathrm{A}}$ und von $L^{*}{ }_{\mathrm{B}}$ in $\mathrm{F}_{\mathrm{B}}, \mathrm{H}_{\mathrm{B}}$ entstehen die Zeitfunktionen $L^{*}{ }_{\mathrm{AF}}, L^{*}{ }_{\mathrm{AH}}$ bzw. $L^{*}{ }_{\mathrm{BF}}, L^{*}{ }_{\mathrm{BH}}$. Der Korrelator $\mathrm{M}_{\mathrm{A}}, \mathrm{S}_{\mathrm{A}}$ verrechnet $L^{*}{ }_{\mathrm{AF}}, L^{*}{ }_{\mathrm{BH}}$ zu dem Zeitmittel $L^{*}{ }_{\mathrm{AF}}, L^{*}{ }_{\mathrm{BH}}$, und der Korrelator $\mathrm{M}_{\mathrm{B}}, \mathrm{S}_{\mathrm{B}}$ die Funktionen $L^{*}{ }_{\mathrm{BF}}, L^{*}{ }_{\mathrm{AH}}$ zu dem Mittel $L^{*}{ }_{\mathrm{BF}}, L^{*}{ }_{\mathrm{AH}}$. Die Korrelations-Ergebnisse werden in einer Subtraktionsinstanz voneinander abgezogen. Das Ergebnis $R$ wird an die Motorik des Tieres weitergeleitet.

\section{Verallgemeinerte lineare Theorie}

Wenn wir von einer linearen Theorie des Bewegungssehens sprechen, so geschieht dies im Hinblick auf die Übertragungs-Eigenschaften der Filter D, F, H und S. Die Nichtlinearität des Zusammenhanges zwischen der Mustergeschwindigkeit $w$ und der optomotorischen Re-

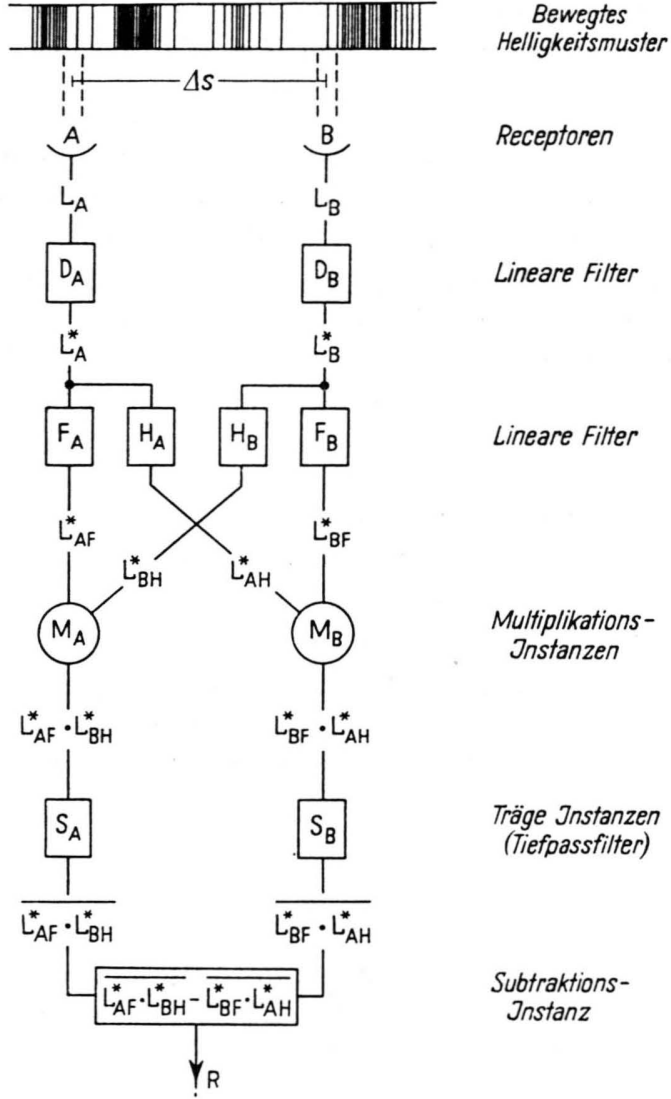

Abb. 1. Verallgemeinerte elementare Funktionsstruktur (Ommatidien-Zweiersystem) der Bewegungsperzeption von Chlorophanus viridis. Gleiche Helligkeitswerte des mit der Geschwindigkeit $w$ bewegten Musters werden von den Ommatidien $\mathrm{A}$ und $\mathrm{B}$ unter der Zeitverschiebung von $\Delta t=\Delta s / w$ aufgenommen. Sie liefern die Informationen für die Nachrichtenkanäle, aus denen die Multiplikatoren $M_{\mathrm{A}}$ und $M_{\mathrm{B}}$ gespeist werden. Die Multiplikations-Instanzen verarbeiten sowohl die Beträge wie auch die Vorzeichen der einlaufenden Signale. Die Leitungen in der Funktionsstruktur stellen daher nicht unmittelbar Nervenbahnen dar, weil deren Fähigkeiten zur Signalübertragung geringer sind.

aktion $R$ ist allein dem Korrelationsprozeß zuzuschreiben.

In dem dieser Arbeit vorausgehenden Artikel $^{5}$ wurden Experimente beschrieben, in welchen sich die schon früher ${ }^{3}$ für wahrscheinlich gehaltene Linearität der zentralnervösen Filter erneut bestätigte. Eine weitere Bestätigung der linearen Übertragungs-Eigenschaften ergibt sich aus optomotorischen Reaktionen auf die Bewegung periodischer Helligkeitsstrukturen, die sich aus mehreren F o u ri e r-Komponenten zusammensetzen: Die Messungen ${ }^{6}$ stimmen mit den berechneten Reaktionsverläufen ${ }^{7}$ überein, die unter der Annahme der Linearität bestimmt wurden.

5 B. Hassenstein, Z. Naturforschg. 14 b, 659 [1959].

${ }^{6}$ B. Hassenstein, Z. vergleich. Physiol. 33, 301 [1951].

7 D. VarJú, Z. Naturforschg. 14 b, [1959], im Druck. 


\section{A. Der Zusammenhang zwischen Mustergeschwin- digkeit $\boldsymbol{w}$ und Reaktion $\boldsymbol{R}$ im Zeitbereich}

Die verallgemeinerte elementare Funktionsstruktur für das Bewegungssehen des Käfers Chlorophanus (Abb. 1) wird von zwei Nachrichtenkanälen gebildet, die miteinander in Wechselwirkung treten. Die optische Umwelt des Tieres stellt die Informationsquelle für beide Nachrichtenkanäle dar.

Die Helligkeitsverteilung denken wir uns aus Helligkeitswerten aufgebaut, die einen Wertebereich $0 \leqq L \leqq L_{\max }$ überstreichen. Bei unregelmäßiger Verteilung der Helligkeitswerte ist $L$ eine Zufallsvariable. Bei der Berechnung sollen verschwindend kleine Aperturen der Rezeptoren vorausgesetzt werden; die Abtastung der Helligkeitswerte soll sich also „punktförmig““ vollziehen *.

Wir betrachten zunächst eine Musterbewegung von links nach rechts (s. Abb. 1). Die Eingangsgrößen $L_{\mathrm{A}}$ und $L_{\mathrm{B}}$ teilen wir auf in ihren Mittelwert $C$ und die Wechselkomponente $G(t)$

$$
L_{\mathrm{A}}=C+G(t), \quad L_{\mathrm{B}}=C+G(t-\Delta t) .
$$

Damit ist $G$ so definiert, daß sein Mittelwert verschwindet. $L_{\mathrm{A}}$ und $L_{\mathrm{B}}$ treten nun in die Filter $\mathrm{D}_{\mathrm{A}}, \mathrm{D}_{\mathrm{B}}$ ein. Da es sich nach den experimentellen Ergebnissen um lineare Filter handelt, können die Zeitfunktionen $L^{*}$ an ihrem Ausgang als Faltungsintegrale ausgedrückt werden. Hierzu denkt man sich die Eingangsfunktion in Impulse zerlegt. Tritt ein Einheitsimpuls ( $\delta$-Funktion) mit den Eigenschaften

$$
\begin{aligned}
\delta\left(t-t_{0}\right) & =\infty \text { für } t=t_{0} \quad \text { jedoch } \int_{-\infty}^{+\infty} \delta\left(t-t_{0}\right) \mathrm{d} t=1 \\
& \text { für } t \neq t_{0}
\end{aligned}
$$

in eines der Filter ein, so erzeugt er am Ausgang des Filters einen Zeitverlauf, den man mit $W\left(t-t_{0}\right)$ bezeichnet. $W$ ist die "Gewichtsfunktion" des Filters. Da wir die Stabilität der Filter voraussetzen dürfen, muß das Integral

konvergieren.

$$
\lim _{T \rightarrow \infty} \int_{-T}^{+T}|W(t)| \mathrm{d} t
$$

Für eine beliebige Eingangsfunktion $F(t)$ erhält man durch „Faltung" das zeitliche Verhalten des Ausganges

$$
F^{*}(t)=\int_{-\infty}^{t} W(t-\xi) F(\xi) \mathrm{d} \xi=\int_{0}^{+\infty} W(\xi) F(t-\xi) \mathrm{d} \xi .
$$

* Daß diese Voraussetzung eine gute Näherung darstellt, geht aus der Analyse der Reaktionen auf periodische Helligkeitsstrukturen ${ }^{7}$ hervor. Danach beträgt der Sehwinkel eines Ommatidiums nur ca. $1^{\circ}$, während der Winkel zwi-
In der verallgemeinerten Funktionsstruktur Abb. 1 liegen je zwei lineare Filter in Serienschaltung. Jedes dieser Doppelfilter besitzt eine Gewichtsfunktion, die sich aus dem Faltungsintegral über die Gewichtsfunktionen der Einzelfilter berechnet ${ }^{8}$. Aus der Symmetrie der optomotorischen Reaktion auf Bewegungen von rechts nach links und von links nach rechts folgt, daß

$$
D_{\mathrm{A}}=D_{\mathrm{B}}=D ; F_{\mathrm{A}}=F_{\mathrm{B}}=F \text { und } H_{\mathrm{A}}=H_{\mathrm{B}}=H
$$

ist. Bezeichnen wir daher mit $W_{(\mathrm{DF})}$ die Gewichtsfunktionen der Längs- und mit $W_{(\mathrm{DH})}$ die Gewichtsfunktionen der Querverbindungen, in Abb. 1, so ergeben sich am Eingang der Multiplikatoren $M_{A}, M_{B}$ die Zeitabhängigkeiten

$$
\begin{aligned}
L^{*}{ }_{\mathrm{AF}} & =\int_{0}^{+\infty} W_{(\mathrm{DF})}(\eta) L_{\mathrm{A}}(t-\eta) \mathrm{d} \eta, \\
L^{*}{ }_{\mathrm{BH}} & =\int_{0}^{+\infty} W_{(\mathrm{DH})}(\xi) L_{\mathrm{B}}(t-\xi) \mathrm{d} \xi, \\
L^{*} \mathrm{BF} & =\int_{0}^{+\infty} W_{(\mathrm{DF})}(\xi) L_{\mathrm{B}}(t-\xi) \mathrm{d} \xi, \\
L^{*} \mathrm{AH} & =\int_{0}^{+\infty} W_{(\mathrm{DH})}(\eta) L_{\mathrm{A}}(t-\eta) \mathrm{d} \eta .
\end{aligned}
$$

Die damit formulierten Eingangsgrößen für die Multiplikations-Instanzen werden dort miteinander multipliziert

$$
\begin{aligned}
& L^{*}{ }_{\mathrm{AF}} \cdot L^{*}{ }_{\mathrm{BH}} \\
& \quad=\int_{0}^{+\infty} W_{(\mathrm{DF})}(\eta) L_{\mathrm{A}}(t-\eta) \mathrm{d} \eta \int_{0}^{+\infty} W_{(\mathrm{DH})}(\xi) L_{\mathrm{B}}(t-\xi) \mathrm{d} \xi .
\end{aligned}
$$

Hierbei kann man $L_{\mathrm{A}}(t-\eta)$ unter das $\xi$-Integral ziehen und erhält

$$
\begin{aligned}
L^{*}{ }_{\mathrm{AF}} \cdot & L^{*{ }_{\mathrm{BH}}} \\
& =\int_{0}^{+\infty} W_{(\mathrm{DF})}(\eta) \mathrm{d} \eta \int_{0}^{+\infty} W_{(\mathrm{DH})} L_{\mathrm{A}}(t-\eta) L_{\mathrm{B}}(t-\xi) \mathrm{d} \xi .
\end{aligned}
$$

Entsprechendes gilt für den Vorgang im Multiplikator $\mathrm{M}_{\mathrm{B}}$. Die Multiplikations-Ergebnisse werden dann in $\mathrm{S}_{\mathrm{A}}$ bzw. in $\mathrm{S}_{\mathrm{B}}$ zeitlich gemittelt. Unter der Voraussetzung, daß $L_{\mathrm{A}}, L_{\mathrm{B}}$ ergodische Zufallsvariable sind, ergibt $\operatorname{sich}^{9}$

schen den Achsen benachbarter Ommatidien $\Delta s=6,8^{\circ}$ ausmacht.

8 Siehe z. B. W. Oppelt, Kleines Handbuch Technischer Regelvorgänge, Verlag Chemie GmbH (1956) S. 44 ff. 


$$
\begin{aligned}
L^{*}{ }_{\mathrm{AF}} \cdot L^{*}{ }_{\mathrm{BH}} & \\
= & \int_{0}^{+\infty} W_{(\mathrm{DF})}(\eta) \mathrm{d} \eta \int_{0}^{+\infty} W_{(\mathrm{DH})}(\xi) L_{\mathrm{A}}(t-\eta) L_{\mathrm{B}}(t-\xi) \mathrm{d} \xi .
\end{aligned}
$$

Der darin enthaltene Ausdruck $\overline{L_{\mathrm{A}}(t-\eta) L_{\mathrm{B}}(t-\xi)}$ entspricht der Kreuzkorrelations-Funktion (KKF) $\Phi_{\mathrm{AB}}$ zwischen $L_{\mathrm{A}}$ und $L_{\mathrm{B}}$. Vereinfachend kann man also schreiben

$\overline{L^{*}{ }_{\mathrm{AF}} \cdot L^{*}{ }_{\mathrm{BH}}}$

$$
=\int_{0}^{+\infty} W_{(\mathrm{DF})}(\eta) \mathrm{d} \eta \int_{0}^{+\infty} W_{(\mathrm{DH})}(\xi) \Phi_{\mathrm{AB}}(\eta-\xi) \mathrm{d} \xi .
$$

Ersetzt man $L_{\mathrm{A}}$ und $L_{\mathrm{B}}$ entsprechend Gl. (1) durch $C+G(t)$ und $C+G(t-\Delta t)$ und subtrahiert gleichzeitig (nach Abb. 1) den vom Korrelator $\mathrm{M}_{B}, \mathrm{~S}_{\mathrm{B}}$ gebildeten Ausdruck $\overline{L^{*}{ }_{\mathrm{BF}} L^{*}{ }_{\mathrm{AH}}}$, so erhält man als Ergebnis für die Reaktion $R$

$$
\begin{gathered}
R=\int_{0}^{+\infty} W_{(\mathrm{DF})}(\eta) \mathrm{d} \eta \int_{0}^{+\infty} W_{(\mathrm{DH})}(\xi) \Phi_{\mathrm{GG}}(\eta-\xi-\Delta t) \mathrm{d} \xi \\
-\int_{0}^{+\infty} W_{(\mathrm{DH})}(\eta) \mathrm{d} \eta \int_{0}^{+\infty} W_{(\mathrm{DF})}(\xi) \Phi_{\mathrm{GG}}(\eta-\xi-\Delta t) \mathrm{d} \xi .
\end{gathered}
$$

Hierin erscheint also anstatt der KKF von Gl. (8 B) die Autokorrelations-Funktion (AKF) $\Phi_{\mathrm{GG}}$ der Umwelthelligkeits-Verteilung. Mit Gl. (9) ist für die Auswerteleistung der Verrechnungsstruktur von Abb. 1 eine mathematische Formulierung gegeben, die - im Unterschied zur früher vorgelegten Ableitung ${ }^{1}$ - für jede Helligkeits-Verteilung der Umwelt, deren $\mathrm{AKF}$ angegeben werden kann, gültig ist.

Nach Gl. (9) bestimmen folgende Größen die Reaktion $R$ :

1. Die Geschwindigkeit $w$ des Helligkeitsmusters, da $\Delta t=\Delta s / w$.

2. Die Struktur der bewegten (ergodischen) Helligkeitsverteilung, ausgedrückt durch die AKF.

3. Die Gewichtsfunktionen der linearen Filter in der Funktionsstruktur.

Der Mittelwert $C$ der Helligkeits-Verteilung fällt durch die Subtraktion der korrelierten Signale heraus, falls er nicht schon durch die - dem Korrelationsproze $§$ vorgeschalteten - linearen Filter, über deren Frequenzgang bisher noch nichts gesagt wurde, entfernt wird.

9 Siehe z. B. I. H. Laning u. R. H. Battin, Random Processes in Automatic Control. McGraw Hill, New York 1956.

10 C. E. Shannon, Proc. Inst. Radio Engr. 37, 10 [1949].
Die Reaktion $R$ ist formal eine transformierte AKF der bewegten Umwelt-Helligkeits-Verteilung.

\section{B. Reaktionen auf spezielle Helligkeits- Verteilungen (Analyse im Zeitbereich)}

Die Definitionsgleichung für die AKF $\Phi_{\mathrm{GG}}$ lautet

$$
\Phi_{\mathrm{GG}}(\xi)=\lim _{T \rightarrow \infty} 1 / 2 T \int_{-T}^{+T} G(t) G(t-\xi) \mathrm{d} t .
$$

$G(t)$ ist im Falle eines Experimentes durch die Umwelt-Helligkeitsverteilung gegeben. Die optomotorische Reaktion $R$ wird als Funktion der Relativgeschwindigkeit zwischen Umwelt und Versuchstier gemessen.

Wir wollen nun aus den experimentellen Ergebnissen die Gewichtsfunktionen der Filter zwischen Rezeptor und Korrelator ermitteln. Eine unentbehrliche Vorarbeit dafür besteht darin, Formulierungen für die später verwendeten speziellen HelligkeitsVerteilungen zu entwickeln:

Eine eindimensionale Helligkeits-Verteilung läßt sich durch drei Größen charakterisieren:

1. Die obere Grenzfrequenz $f_{\max }$, die man zu einer Fourier-Darstellung der Verteilung (bei Vorgabe einer Approximations-Genauigkeit) benötigt.

2. Die durchschnittlich in der Verteilung auftretenden Helligkeits-Unterschiede (Kontraststärke).

3. Gesetzmäßigkeiten, welche die Aufeinanderfolge der einzelnen Helligkeitswerte kontrollieren.

1. In einer Orts- oder Zeitfunktion ist nach SHaNNon ${ }^{10}$ die örtliche $(\Delta x)$ bzw. zeitliche $(\Delta t)$ Ausdehnung des Elementarbereiches, innerhalb dessen sich ein vom Nachbarsignal unterscheidbares Signal definieren läßt, durch die obere Grenzfrequenz der Verteilung festgelegt. Im vorliegenden Problem ist die zeitliche Breite eines Elementarbereiches der $G$-Funktion von der oberen Grenzfrequenz in der räumlichen Helligkeits-Verteilung sowie von der Bewegungsgeschwindigkeit des Musters abhängig. Der Zusammenhang zwischen $\Delta t$ und $\Delta x$ ist dabei

$$
\Delta t=\Delta x / w .
$$

Verdoppelung der Geschwindigkeit zieht also Halbierung des $\mathrm{Sh}$ annon-Intervalles im Zeitbereich nach sich.

2. Als Maß für die durchschnittliche Kontraststärke verwenden wir die mittlere quadratische Streuung der Helligkeitswerte $L$ um ihren Mittelwert $C$. Ist die Helligkeitsverteilung statistischer Natur und bezeichnet man mit $p(L)$ die Wahrscheinlichkeitsdichte für die Werteverteilung, dann ist $p(L) \mathrm{d} L$ die Wahrscheinlichkeit dafür, daß ein Helligkeitswert im Bereich zwischen $L$ und $L+\mathrm{d} L$ liegt. Die mittlere quadratische Streuung der 
Helligkeitswerte ist definiert als

$$
\sigma^{2}=(L-C)^{2}=\int_{0}^{L_{\max }}(L-C)^{2} p(L) \mathrm{d} L=\overline{L^{2}}-C^{2} .
$$

Ist die Helligkeits-Verteilung ergodisch, so ändert sich $p(L)$ nicht mit dem Ort. $\sigma^{2}$ läßt sich unter dieser Voraussetzung auch als quadratisches Zeitmittel von $G(t)$, der Wechselkomponente der Verteilung, berechnen.

$$
\sigma^{2}=\lim _{T \rightarrow \infty} 1 / 2 T \int_{-T}^{+T} G^{2}(t) \mathrm{d} t .
$$

Bei der folgenden Untersuchung beschränken wir uns stets auf ergodische Verteilungen. Ist die HelligkeitsVerteilung analytisch beschreibbar, so kann $\sigma^{2}$ aus Gl. (12 B) bestimmt werden.

3. Die dritte für eine Verteilung charakteristische Größe betrifft das Anordnungsprinzip für die einzelnen Helligkeitswerte. Eine extreme Möglichkeit ist die der reinen Zufallsverteilung (Helligkeits-Rauschfunktion), d. h. zwischen benachbarten Werten besteht keine Korrelation. In diesem Fall besitzt die AKF der Wechselkomponente $G$ nur im „Punkte Null“ der Zeitverschiebung $\xi$ einen von null verschiedenen Wert. Helligkeits-Rauschfunktionen werden vollständig durch die erste Wahrscheinlichkeitsdichte-Funktion $p(L)$ beschrieben.

Ein entgegengesetzter Grenzfall wäre zum Beispiel eine periodische Verteilung der Helligkeitswerte, am einfachsten verwirklicht in einer sinusförmigen Helligkeits-Verteilung. Beide Grenzfälle sind experimentell so weit wie möglich angenähert worden. Sie sollen zunächst in Form ihrer AKF in die allgemeine Reaktionsgleichung eingesetzt und die Ergebnisse mit den empirisch gefundenen verglichen werden.

B. $\alpha$. Verarbeitung bewegter Helligkeits-Rauschfunktionen mit Tiefpa $\beta$ - Filtern

Die AKF von Rauschfunktionen mit dem konstanten Amplitudenspektrum $\sigma$ ist eine $\delta$-Funktion mit dem Faktor $\sigma^{2}$. Führt man sie in Gl. (9) ein, so ergibt sich für die optomotorische Reaktion $R$

$$
R=\sigma^{2} \int_{\eta=\Delta t}^{+\infty} W_{(\mathrm{DF})}^{W_{(\mathrm{DF})}(\eta)} W_{(\eta t)}^{W_{(\mathrm{DH})}(\eta)} W_{(\mathrm{DH})}^{(\eta-\Delta t)} \mathrm{d} \eta .
$$

Betrachten wir zunächst die Filterkombinationen (DF) und (DH) als Tiefpässe mit den Zeitkonstanten $\tau_{\mathrm{F}}$ und $\tau_{\mathrm{H}}$. Unter dieser Voraussetzung erhält man für die Gewichtsfunktionen

$$
\begin{array}{rlrl}
W_{(\mathrm{DF})}=\frac{1}{\tau_{\mathrm{F}}} e^{-t / \tau_{\mathrm{F}}} & t>0, \\
W_{(\mathrm{DH})}=\frac{1}{\tau_{\mathrm{H}}} e^{-t / \tau_{\mathrm{II}}} & t>0 .
\end{array}
$$

Die Kinetik solcher Filter entspricht physikochemisch einer Reaktion erster Ordnung. Führt man die Gewichtsfunktionen aus Gl. (14) in Gl. (13) ein, so ergibt sich nach einfacher Zwischenrechnung

$$
R=\frac{\sigma^{2}}{\tau_{\mathrm{F}}+\tau_{\mathrm{H}}}\left[e^{-1 / z}-e^{-\alpha / z}\right]
$$

mit $z=\tau_{\mathrm{F}} w / \Delta s$ und $\alpha=\tau_{\mathrm{F}} / \tau_{\mathrm{H}}$.

In einer früheren Arbeit ${ }^{4}$ wurden Messungen der optomotorischen Reaktion auf die Bewegung statistisch verteilter Helligkeitswerte beschrieben. Das experimentell verwendete Helligkeitsmuster stellte eine erste Approximation an den Grenzfall des „weißen“ Rauschens dar, weil die Breite der einzelnen Helligkeitswerte kleiner als die Länge der Basis $\Delta s$ zwischen den Elementen $\mathrm{A}$ und $\mathrm{B}$ gewählt wurde. Die Versuchsergebnisse $(R=$ Funktion der Mustergeschwindigkeit $w$ ) stimmten gut mit dem von Gl. (15) geforderten Verlauf überein. Für die optimal an die experimentellen Daten angepaßte Reaktionskurve ergaben sich die Zeitkonstanten $\tau_{\mathrm{F}}=3,5 \mathrm{sec}$, $\tau_{\mathrm{H}}=0,046 \mathrm{sec}$ und entsprechend $\alpha=76,24$.

$$
\begin{gathered}
\text { B. } \beta \text {. Verarbeitung bewegter } \\
\text { sinusförmiger Helligkeits- } \\
\text { Verteilungen mit Tiefpaßiltern }
\end{gathered}
$$

Eine sinusförmige Helligkeits-Verteilung ist gegeben durch

$$
L=C+A \sin (k x-\varphi) \quad \text { mit } \quad A \leqq C .
$$

$C$ ist wiederum der Mittelwert von $L, A$ die Helligkeits-Amplitude der Wechselkomponente, $k$ die Wellenzahl $2 \pi / \lambda, \lambda$ die Wellenlänge und $\varphi$ ein Phasenwinkel. Die AKF der Funktion nach Gl. (16) ist

$$
\begin{gathered}
\Phi(x)=C^{2}+\left(A^{2} / 2\right) \cos k x^{\prime} \\
x^{\prime} \text { ist ein Ortsintervall }
\end{gathered}
$$

und dementsprechend die AKF $\Phi_{\mathrm{GG}}$ der Zeitabhängigkeit für die reine Wechselkomponente in den Elementen $A, B$

$$
\Phi_{\mathrm{GG}}(\xi)=\sigma^{2} \cos k w \xi,
$$

wobei $\sigma^{2}=A^{2} / 2, w$ der Geschwindigkeit des Musters und $\xi$ der in Gl. (10) verwendeten Bezeichnung für ein Zeitintervall entspricht.

Bewegt man die periodische Helligkeits-Verteilung Gl. (16) relativ zu den Eingängen der Funktionsstruktur und setzt man wieder entsprechend Gl. (14) als Gewichtsfunktionen der Filterkombinationen diejenige von Tiefpässen ein, so erhält man für die Reaktion 


$$
\begin{aligned}
R= & \int_{\eta=0}^{+\infty} \frac{1}{\tau_{\mathrm{F}}} e^{-\left(1 / \tau_{\mathrm{F}}\right) \eta} \mathrm{d} \eta \int_{\xi=0}^{+\infty} \frac{1}{\tau_{\mathrm{H}}} e^{-\left(1 / \tau_{\mathrm{H}}\right) \xi} \sigma^{2} \cos k w(\eta-\xi-\Delta t) \mathrm{d} \xi \\
& -\int_{\eta=0}^{+\infty} \frac{1}{\tau_{\mathrm{H}}} e^{\left(1 / \tau_{\mathrm{H}}\right) \eta} \mathrm{d} \eta \int_{\xi=0}^{+\infty} \frac{1}{\tau_{\mathrm{F}}} e^{\left(1 / \tau_{\mathrm{F}}\right) \xi} \sigma^{2} \cos k w(\eta-\xi-\Delta t) \mathrm{d} \xi .
\end{aligned}
$$

Die Auswertung liefert

$$
R=[\sin (k \Delta s)]_{\left[\left(1 / \tau_{\mathrm{F}}^{2}\right)+k^{2} w^{2}\right]\left[\left(1 / \tau_{\mathrm{H}^{2}}\right)+k^{2} w^{2}\right]}
$$

Hierin ist $\Delta s$ wie in Abb. 1 der räumliche Abstand zwischen den Eingangsinstanzen $A$ und $B$.

Nach Gl. (19 B) setzt sich $R$ aus zwei Anteilen zusammen, die multiplikativ miteinander verknüpft sind. Der erste von $w$ und allen Zeitkonstanten des Systems unabhängige Anteil $\sin (k \Delta s)$ ist periodischer Natur, d. h. er wechselt mit variierendem $\lambda$ (Wellenlänge des periodischen Musters) periodisch zwischen positiven und negativen Werten, so daß $R$ nur bereichsweise (für positive Werte) eine zutreffende Information über die Bewegungsrichtung des Helligkeits-Verlaufes liefert. Dieses Phänomen wurde von HerTz ${ }^{11}$ aus einfachen geometrischen Überlegungen gefordert und von $\mathrm{G}_{\mathrm{AvEL}}{ }^{12}$ und $\mathrm{H}_{\text {ASSENSTEIN }}{ }^{6}$ experimentell untersucht und bestätigt. Eine quantitative Behandlung ${ }^{7}$ dieser Erscheinungen der "geometrischen Interferenz" auf der Basis von Gl. (19 B) führte zur Übereinstimmung mit den experimentellen Befunden, wenn die im zweiten Teil dieser Gleichung auftretenden Größen $k$ und $w$ im Experiment nicht variiert wurden.

Der geschwindigkeitsabhängige zweite Anteil von $R$ in Gl. (19 B) hat die Form eines gebrochenen rationalen Ausdruckes. In Abb. 2 wurde dieser Anteil unter Einsetzen der früher gewonnenen Zahlenwerte für $\tau_{\mathrm{F}}$ und $\tau_{\mathrm{H}}$ aufgetragen (ausgezogene Kurve). Um diesen theoretischen Verlauf experimentell nachzuprüfen, wurde eine sinusförmige Helligkeits-Verteilung mit der Wellenlänge $\lambda=4,7 \Delta s$ vor dem Ommatidienraster des Käfers Chlorophanus viridis bewegt und die hierauf erfolgende optomotorische Geschwindigkeit gemessen ${ }^{5}$. Das experimentelle Ergebnis zeigt die rechte Kurve der Abb. 2. Sie weicht von dem unter Voraussetzung von Tiefpaßfiltern ermittelten Reaktionsverlauf erheblich ab: Sie ist um den Faktor 10 zu höheren Geschwindigkeiten verschoben und erstreckt sich über einen größeren Geschwindigkeitsbereich.

Die Abweichungen zwischen der experimentell aufgenommenen und der theoretisch bestimmten Reak-

11 M. Hertz, Z. vergleich. Physiol. 21, 579 [1934].

12 L. von Gavel, Z. vergleich. Physiol. 27, 80 [1939].

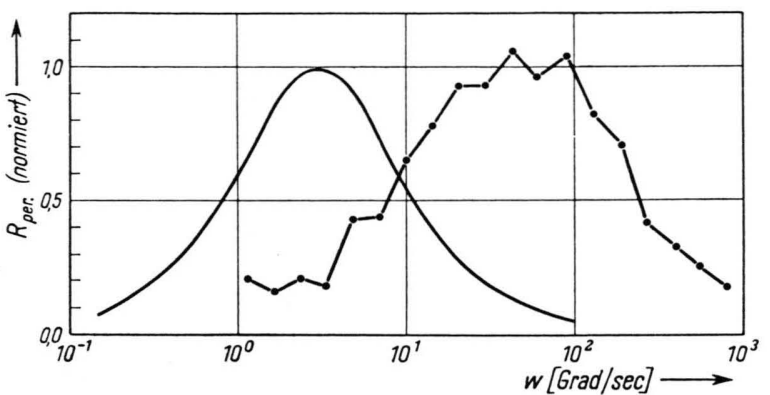

Abb. 2. Die Reaktionsgröße der Funktionsstruktur mit Tiefpässen (linke Kurve) und die optomotorische Reaktion des Käfers (rechte Kurve) als Funktion der Geschwindigkeit eines sinusförmigen Helligkeitsmusters mit der Wellenlänge $\lambda=4,7 \Delta s$. Meßwerte nach Hassenstein ${ }^{5}$.

tionskurve lassen darauf schließen, daß die Signalübertragung von den Eingängen A, B zu den Korrelatoren durch Tiefpässe [s. Gl. (14)] nicht richtig oder nicht vollständig wiedergegeben wird. Dieser Befund macht es notwendig, von der speziellen Vorstellung der Übertragung durch Tiefpaßfilter noch einmal auf die allgemeingültige Gl. (9) zurückzugehen, und die Analyse an der Reaktion auf sinusförmige Helligkeits-Verteilung anzusetzen. Es ist zu erwarten, daß die Bestimmung der ÜbertragungsEigenschaften der Filter auf dieser Grundlage mit größerer Sicherheit durchgeführt werden kann, als auf der Grundlage der Reaktion auf unregelmäßige Helligkeitsmuster. Dafür lassen sich drei Gründe anführen: 1. Die endliche Apertur der Ommatidien verfälscht die sinusförmige Helligkeits-Verteilung der zugrunde gelegten Wellenlänge $\lambda=4,7 \Delta s=66^{\circ}$ so gut wie gar nicht, während sie im Frequenzspektrum statistisch zusammengesetzter Helligkeitsmuster die höheren Frequenzen unterdrückt ${ }^{7}$. 2. Die im Experiment verwandte sinusförmige Helligkeits-Verteilung entspricht dem in die Rechnung eingesetzten idealen Sinusvorgang viel besser, als der aus $7^{\circ}$ breiten Graustreifen zusammengesetzte Streifenzylinder ${ }^{4}$ einer Helligkeits-Rauschfunktion mit konstantem Amplitudenspektrum. 3. Eine ideale Helligkeits-Rauschverteilung müßte sämtliche Frequenzen (von null bis unendlich) mit gleicher Amplitude enthalten. Wenn eine statistische Helligkeitsverteilung - wie in den 
Experimenten von Hassenstein - aber auf einem Zylinder aufgetragen ist, fehlen ihr die tiefen Frequenzen, da sich das Muster nach einem Umlauf von $360^{\circ}$ wiederholt.

Für die folgende Analyse mit sinusförmiger Helligkeitsverteilung überträgt man Gl. (9) in den Frequenzbereich, weil sich dann die Bestimmung der Gewichtsfunktion bzw. der ihr äquivalenten Übertragungs-Funktionen mathematisch einfacher gestaltet. Die fouriertransformierte AKF der sinusförmigen Helligkeits-Verteilung ist eine $\delta$-Funktion, unter deren Einwirkung die Integration der Reaktionsgleichung unmittelbar ausgeführt werden kann.

\section{Der Zusammenhang zwischen Mustergeschwin- digkeit $\boldsymbol{w}$ und Reaktion $\boldsymbol{R}$ im Frequenzbereich}

Die Reaktion $R=R(\Delta t)$ ist formal eine transformierte Korrelationsfunktion. Die ihr zugeordnete Spektraldichte - wir bezeichnen sie mit $Z(i \omega)$ ergibt sich aus einer Fourier-Transformation ${ }^{9}$.

$Z(i \omega)=\frac{1}{\pi} \int_{-\infty}^{+\infty} R(\Delta t) e^{-i \omega \Delta t} \mathrm{~d} \Delta t \quad i=V-1$.

Führt man für $R(\Delta t)$ den in Gl. (9) angegebenen Ausdruck ein, so ergibt sich

$$
\begin{aligned}
Z(i \omega)= & {\left[\int_{\eta=0}^{+\infty} W_{(\mathrm{DF})}(\eta) e^{-i \omega \eta} \mathrm{d} \eta \int_{\xi=0}^{+\infty} W_{(\mathrm{DH})}(\xi) e^{+i \omega \xi} \mathrm{d} \xi\right.} \\
& \left.-\int_{\eta=0}^{+\infty} W_{(\mathrm{DH})}(\eta) e^{-i \omega \eta} \mathrm{d} \eta \int_{\xi=0}^{+\infty} W_{(\mathrm{DF})}(\xi) e^{+i \omega \xi} \mathrm{d} \xi\right] \frac{1}{\pi} \int_{-\infty}^{+\infty} \Phi_{\mathrm{GG}}(\eta-\xi-\Delta t) e^{-i \omega(-\eta+\xi+\Delta t)} \mathrm{d} \Delta t .
\end{aligned}
$$

Setzen wir $\tau=-\eta+\xi+\Delta t$ und berücksichtigen die Symmetrieeigenschaften der AKF $[\Phi(-\tau)=\Phi(\tau)]$, dann erhält man für das Integral über $\Phi_{\mathrm{GG}}$

$$
S(\omega)=\frac{1}{\pi} \int_{-\infty}^{+\infty} \Phi(\tau) e^{-i \omega \tau} \mathrm{d} \tau .
$$

Hierin bedeutet $S(\omega)$ die Spektraldichte von $G(t)$. Die in dem Klammerausdruck von Gl. (20 B) stehenden F o u r i e r - Transformationen der Gewichtsfunktionen ergeben die Übertragungsfunktionen $Y(i \omega)$ der Filterkombinationen. Es ist also

$$
\begin{gathered}
Y_{(\mathrm{DF})}(i \omega)=\int_{\eta=0}^{+\infty} W_{(\mathrm{DF})}(\eta) e^{-i \omega \eta} \mathrm{d} \eta, \\
Y^{*}{ }_{(\mathrm{DH})}(i \omega)=\int_{\xi=0}^{+\infty} W_{(\mathrm{DH})}(\xi) e^{+i \omega \xi} \mathrm{d} \xi, \\
Y_{(\mathrm{DH})}(i \omega)=\int_{\eta=0}^{+\infty} W_{(\mathrm{DH})}(\eta) e^{-i \omega \eta} \mathrm{d} \eta, \\
Y^{*}{ }_{(\mathrm{DF})}(i \omega)=\int_{\xi=0}^{+\infty} W_{(\mathrm{DF})}(\xi) e^{+i \omega \xi} \mathrm{d} \xi .
\end{gathered}
$$

Hierbei ist $Y^{*}$ die konjugierte komplexe Übertragungsfunktion. Gl. (20 B) läßt sich jetzt zusammenfassen

$$
Z(i \omega)=\left|\begin{array}{ll}
Y_{(\mathrm{DF})} & Y^{*}(\mathrm{DF}) \\
Y_{(\mathrm{DH})} & Y^{*}{ }_{(\mathrm{DH})}
\end{array}\right| S(\omega) .
$$

Die in der Determinante stehenden Übertragungs- funktionen charakterisieren die Filterkombinationen (DF) und (DH) eindeutig. Sie setzen sich multiplikativ aus den Übertragungsfunktionen der Einzelfilter $\mathrm{D}$ und $\mathrm{F}$ bzw. $\mathrm{D}$ und $\mathrm{H}$ zusammen ${ }^{8}$. Infolgedessen läßt sich für Gl. (20 C) auch schreiben

$$
Z(i \omega)=Y_{\mathrm{D}} Y^{*}{ }_{\mathrm{D}}\left|\begin{array}{cc}
Y_{\mathrm{H}} & Y^{*}{ }_{\mathrm{H}} \\
Y_{\mathrm{F}} & Y^{*}{ }_{\mathrm{F}}
\end{array}\right| S(\omega) .
$$

Die Reaktion $R$ ergibt sich aus Gl. (20 D) durch eine $\mathrm{F}$ o u r i e r - Rücktransformation ${ }^{9}$

$R(\Delta t)=\frac{1}{2} \int_{-\infty}^{+\infty} Y_{\mathrm{D}} Y^{*}{ }_{\mathrm{D}}\left|\begin{array}{ll}Y_{\mathrm{F}} & Y^{*}{ }_{\mathrm{F}} \\ Y_{\mathrm{H}} & Y^{*}{ }_{\mathrm{H}}\end{array}\right| S(\omega) e^{+i \omega \Delta t} \mathrm{~d} \omega$.

Diese Gleichung entspricht in ihrer Aussage der Gl. (9), enthält aber an Stelle der Gewichtsfunktionen $W(t)$ der Filter deren Frequenz-Übertragungsfunktionen $Y$.

$$
\text { C. } \alpha . \text { Helligkeitsverteilung: }
$$

Sinusförmige Periodizität (Analyse i m Frequenzbereich).

Um die Übertragungs-Eigenschaften der Filter D, $\mathrm{F}$ und $\mathrm{H}$ quantitativ ermitteln zu können, betrachten wir nochmals den Fall einer sinusförmigen Helligkeitsverteilung. Für die Spektraldichte $S(\omega)$ der reinen Wechselkomponente $G(t)$ dieser Verteilung erhält man unter Berücksichtigung der Gln. (16), (18) und $(20 \mathrm{~A})$

$$
S(\omega)=\sigma^{2}[\delta(\omega-k w)+\delta(\omega+k w) .
$$


$S(\omega)$ setzt sich also aus den beiden Spektrallinien $\omega= \pm k w$ zusammen, die mit der Stärke $\sigma^{2}$ auftreten. Trägt man Gl. (24) in Gl. (23) ein, so ergibt sich schließlich für $R(\Delta t)$

$$
R=i\left|Y_{\mathrm{D}}\right|^{2}\left|\begin{array}{ll}
Y_{\mathrm{F}} & Y^{*} \mathrm{~F} \\
Y_{\mathrm{H}} & Y^{*} \mathrm{H}
\end{array}\right| \sin (k w \Delta t) \cdot \sigma^{2} .
$$

Obwohl in vorstehender Gleichung noch keine speziellen Annahmen - außer der Linearität - über die Filter enthalten sind, tritt das experimentell verifizierte "Interferenzglied“ $\sin (k w \Delta t)$ auf. Es ist also von den Übertragungs-Eigenschaften und den Zeitkonstanten der Filter unabhängig.

Abb. 3 enthält die experimentellen Meßergebnisse des Zusammenhanges $R=$ Funktion der Mustergeschwindigkeit $w$ für den Fall einer sinusförmigen Helligkeits-Verteilung mit der Wellenlänge $\lambda=4,7 \Delta s . \sin (k w \Delta t)$ ist konstant, und zwar annähernd +1 , so daß die optomotorische Reaktion nur noch von den Übertragungsfunktionen $Y_{\mathrm{D}}, Y_{\mathrm{F}}$ und $Y_{\mathrm{H}}$ abhängt. Die Aufgabe besteht darin, diese Übertragungsfunktionen so zu bestimmen, daß die Meßergebnisse durch die Beziehung

$$
R_{\lambda=4 \Delta s}=i\left|Y_{\mathrm{D}}\right|^{2}\left|\begin{array}{cc}
Y_{\mathrm{F}} & Y^{*} \mathrm{~F} \\
Y_{\mathrm{H}} & Y^{*}{ }_{\mathrm{H}}
\end{array}\right| \cdot \sigma^{2}
$$

möglichst gut wiedergegeben werden.

Das zeitliche Verhalten aller Filterausgänge auf eine Störung am Filtereingang muß durch lineare Differentialgleichungen mit konstanten Koeffizienten beschrieben werden, weil die Übertragungs-Eigenschaften der Filter erstens linear sind und zweitens sich zeitlich nicht ändern. Die Gleichungen können gewöhnlicher oder auch partieller Art sein.

Wir machen zunächst die Annahme, die Filter könnten durch gewöhnliche Differentialgleichungen beschrieben werden. Für die Gewichtsfunktion $W$ eines Filters ergibt sich unter dieser Voraussetzung die allgemeine Bestimmungsgleichung ${ }^{13}$

$$
\left(\sum_{\nu=0}^{N} a_{\nu} \frac{d^{\nu}}{\mathrm{d} t^{\nu}}\right) W(t)=\left(\sum_{\mu=0}^{M} b_{u} \frac{d^{\mu}}{\mathrm{d} t^{\mu}}\right) \delta(t) .
$$

Hierin sind $a_{v}, b_{\mu}$ konstante Koeffizienten. $M$ kann höchstens gleich $N$ werden, weil sonst die Stabilitätsbedingung $\mathrm{Gl}$. (3) verletzt wird. Für verschwindende Anfangswerte im Zeitpunkt $t=0$ erhält man durch F o urier-Transformation von Gl. (26) die der Gewichtsfunktion im Zeitbereich zugeordnete Über-

13 Siehe z. B. H. M. James u. P. R. Weiss, Theory of Servomechanisms, McGraw Hill, New York 1949 (Radiation Laboratory Series No. 25).

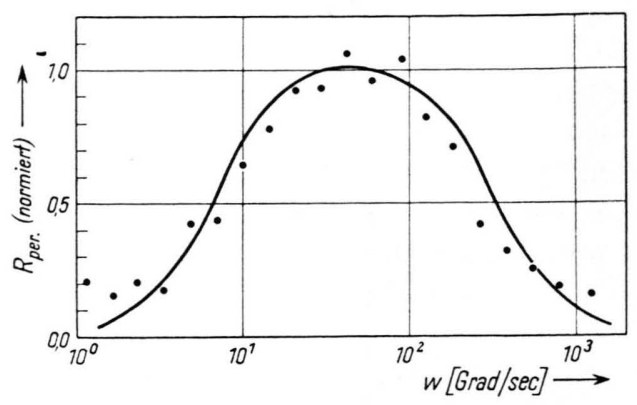

Abb. 3. Die Reaktionsgröße der verallgemeinerten Funktionsstruktur (ausgezogene Kurve) und die optomotorische Reaktion des Käfers (Meßpunkte) als Funktion der Geschwindigkeit eines sinusförmigen Helligkeitsmusters mit der Wellenlänge $\lambda=4,7 \Delta s$. Meßwerte nach Hassenstein ${ }^{5}$.

tragungsfunktion im Frequenzbereich

$$
Y(p)=\frac{\sum_{\mu=0}^{M} b_{\mu} p^{\mu}}{\sum_{\nu=0}^{N} a_{\nu} p^{\nu}} \quad p=i \omega .
$$

Bezeichnet man das Zählerpolynom in Gl. (27) mit $Y_{a}$ und das Nennerpolynom mit $Y_{\beta}$ und führt man diesen allgemeinen Ausdruck für die Übertragungsfunktion in Gl. (25 B) ein, so ergibt sich

$$
R_{\lambda=4 \Delta s}=i\left|\frac{Y_{\mathrm{D} a}}{Y_{\mathrm{D} \beta}}\right|^{2}\left|\begin{array}{cc}
\frac{Y_{\mathrm{F} a}}{Y_{\mathrm{F} \beta}} & Y_{\mathrm{F} \alpha}^{*} \\
\frac{Y_{\mathrm{H} \alpha}^{*}}{Y_{\mathrm{H} \beta}} & \frac{Y^{*} \mathrm{H} a}{Y_{\mathrm{H} \beta}^{*}}
\end{array}\right| \cdot \sigma^{2}
$$

und nach Zwischenrechnung schließlich

$$
\begin{aligned}
R_{\hat{\lambda}=4 \Delta s=-2 \sigma^{2}}\left|\frac{Y_{\mathrm{D} a}}{Y_{\mathrm{D} \beta}}\right|^{2} \\
\cdot \frac{\text { Imaginärteil von }\left(Y_{\mathrm{F} \alpha} Y_{\mathrm{F} \beta}^{*} Y_{\mathrm{H} \alpha}^{*} Y_{\mathrm{H} \beta}\right)}{\left|Y_{\mathrm{F} \beta}\right|^{2}\left|Y_{\mathrm{H} \beta}\right|^{2}}
\end{aligned}
$$

Zähler und Nenner sind in vorstehender Gleichung Polynome in $k w$. Mit Hilfe einer einfachen Überlegung läßt sich zeigen, daß der Imaginärteil des Polynomproduktes $\left(Y_{\mathrm{F} a} Y_{\mathrm{F} \beta}^{*} Y^{*} \mathrm{H} a Y_{\mathrm{H} \beta}\right)$ nur ungerade Potenzen von $k w$ enthält, während die $\mathrm{Ab}$ solutquadrate der Polynome $Y_{\mathrm{F} \beta}$ und $Y_{\mathrm{H} \beta}$ im Nenner nur gerade Potenzen in $k w$ aufweisen. Da auch die Absolutquadrate von $Y_{\mathrm{D} \alpha}$ und $Y_{\mathrm{D} \beta}$ nur aus geraden Potenzen bestehen, enthält also der Zähler in Gl. (25 D) ungerade und der Nenner gerade Potenzen von $k w$. Gl. (25 D) beschreibt die Beziehung zwischen der Reaktion $R$ und der Geschwindigkeit $w$ der sinusförmigen Helligkeits-Verteilung für den Fall, daß die Übertragungs-Eigenschaften der Filter 
durch gewöhnliche Differentialgleichungen beschrieben werden können.

Die Aufgabe ist jetzt zu entscheiden, ob die experimentell ermittelte Reaktionskurve (s. Meßwerte in Abb. 3) in ihrem Verlauf und ihren Vorzeichenverhältnissen auf der Basis von Gl. (25 D) approximiert werden kann. Wir betrachten eine Annäherung als optimal, wenn ein Reaktionsverlauf angegeben werden kann, der erstens innerhalb der mittleren quadratischen Streuung der Meßwerte verläuft und zweitens eine möglichst kleine Zahl von Parametern (Zeitkonstanten) enthält.

Aus Gl. (25 D) läßt sich entnehmen, daß a) das Zählerpolynom stets nur ungerade, das Nennerpolynom nur gerade Potenzen von $k w$ enthält. Die Stabilitätsbedingung Gl. (3) für die Filter fordert, daß der Grad von $Y_{\mathrm{F} \alpha}, Y_{\mathrm{H} \alpha}$ bzw. $Y_{\mathrm{D} \alpha}$ höchstens gleich dem Grad der entsprechenden Polynome $Y_{\mathrm{F} \beta}, Y_{\mathrm{H} \beta}$ bzw. $Y_{\mathrm{D} \beta}$ sein kann. In Kombination mit a folgt daraus, daß b) der Grad des Zählerpolynoms in Gl. (25 D) kleiner als der Grad des Nennerpolynoms sein muß. Aus der Reaktionskurve in Abb. 3 sind vier Bedingungen zu entnehmen, die von Gl. (25 D) erfüllt werden müssen: $\alpha$ ) Die Reaktionskurve darf im Bereich $0<k w<+\infty$ nur ein Maximum durchlaufen. $\beta$ ) Sie muß zu diesem Maximum - im halblogarithmischen Maßstab - symmetrisch verlaufen und $\gamma$ ) eine Halbwertsbreite (in diesem Maßstab) von ca. 50 Einheiten besitzen. $\delta$ ) Ein Vorzeichenwechsel von $w$ muß einen Vorzeichenwechsel von $R$ nach sich ziehen.

Um festzustellen, ob Gl. (25 D) diese vier Bedingungen erfüllen kann, hat man ausgehend von der einfachsten Möglichkeit systematisch den Grad von Zähler- und Nennerpolynom zu erhöhen und für jeden gewählten rationalen Ausdruck die Koeffizienten in den Polynomen so zu variieren, daß die Reaktionskurve möglichst approximiert wird. Das Hinzufügen eines neuen Gliedes - sowohl im Zähler als auch im Nennerpolynom - erhöht dabei jeweils die Zahl der Koeffizienten (Zeitkonstanten) um eins. Wir haben zunächst sämtliche Kombinationsmöglichkeiten von drei Koeffizienten daraufhin untersucht, ob sie im Rahmen von Gl. (25 D) den Bedingungen $\alpha-\delta$ genügen. Es zeigte sich ${ }^{*}$, daß stets mindestens eine der drei Forderungen $\alpha-\gamma$ nicht zu erfüllen ist, wie auch immer die Koeffizienten gewählt werden.

* Bis auf eine Kombinationsmöglichkeit konnte analytisch streng bewiesen werden, daß Gl. (25 D) den gestellten Forderungen nicht genügt. Für die letzte Kombinations-
Eine gute Approximation der in Abb. 3 dargestellten Reaktionskurve ergibt sich jedoch, wenn der Zähler des gebrochenen rationalen Ausdruckes in Gl. (25 D) nur gerade Potenzen von $k w$ enthält. In diesem Fall läßt sich eine Lösung mit sogar nur zwei Koeffizienten angeben. Sie ist

$$
R_{\lambda=4 \Delta s}=B \frac{k^{2} w^{2}}{\left(a_{0} \mathrm{~F}^{2}+k^{2} w^{2}\right)\left(a_{0 \mathrm{H}^{2}}+k^{2} w^{2}\right)},
$$

wobei die Konstanten $a_{0 \mathrm{~F}}, a_{0 \mathrm{H}}$ die Werte $a_{0 \mathrm{~F}}=0,62$, $a_{0 \mathrm{H}}=31,2$ annehmen. $B$ ist eine Proportionalitätskonstante. Gl. (26 A) ist in Abb. 3 (ausgezogene Kurve) dargestellt. Diese Gleichung widerspricht aber erstens der Forderung a, zweitens der Forderung $\delta$. Will man mit Hilfe von Gl. (26 A) trotzdem sowohl den Reaktionsverlauf in Abb. 3 approximieren als auch der Forderung $\delta$ nach dem Vorzeichenwechsel in $R$ nachkommen, so muß sie lauten

$$
R_{\lambda=4 \Delta s}=\frac{B_{1}|k w| B_{2} k w}{\left(a_{0} \mathrm{~F}^{2}+k^{2} w^{2}\right)\left(a_{0} \mathrm{H}^{2}+k^{2} w^{2}\right)}
$$

mit $B_{1} \cdot B_{2}=B$.

Wenn wir die vorstehende Gleichung als das Produkt zweier Reaktionsanteile auffassen, so kann der eine

$$
\frac{B_{2} k w}{\left(a_{0 \mathrm{~F}}{ }^{2}+k^{2} w^{2}\right)\left(a_{0 \mathrm{H}^{2}}+k^{2} w^{2}\right)}
$$

dem zweiten Teil von Gl. (25 D) zugeschrieben werden, da er im Zähler nur ungerade und im Nenner nur gerade Potenzen von $k w$ enthält und das Nennerpolynom von höherem Grade als das Zählerpolynom ist. Der zweite Faktor

$$
B_{1}|k w|
$$

entspricht dem ersten Anteil von GI. (25 D) nicht, da $\left|\frac{Y_{\mathrm{D} \alpha}}{Y_{\mathrm{D} \beta}}\right|^{2}$ auf der Basis gewöhnlicher Differentialgleichungen nur gerade Potenzen von $k w$ enthält. Es kann also nur der eine Teil des Übertragungsprozesses auf der Basis linearer gewöhnlicher Differentialgleichungen behandelt werden, wenn wir eine Minimallösung suchen, d. h. die Zahl der Koeffizienten auf ein Mindestmaß beschränken ${ }^{14}$. Es bleibt zu untersuchen, ob der Übertragungsfaktor $B_{1}|k w|$ durch lineare partielle Differentialgleichungen zu beschreiben ist.

Wir betrachten zunächst den Reaktionsanteil, der dem zweiten Teil von Gl. (25 D) entspricht. Durch Nennervergleich zwischen den Gl. (26 C) und (25 D)

möglichkeit haben wir mit Hilfe eines numerischen Verfahrens dasselbe Ergebnis gefunden.

14 B. Hassenstein u. W. Reichardt, Z. Naturforschg. 8 b, 518 [1953]. 
erhält man

$\left|Y_{\mathrm{F} \beta}\right|^{2}\left|Y_{\mathrm{H} \beta}\right|^{2}=\left(a_{0 \mathrm{~F}}^{2}+k^{2} w^{2}\right)\left(a_{0 \mathrm{H}^{2}}+k^{2} w^{2}\right)$.

Da $Y_{\mathrm{F} \beta}$ und $Y_{\mathrm{H} \beta}$ Polynome von $k w$ sind, lassen sich zwei Lösungen für vorstehende Gleichung finden:

a) $Y_{\mathrm{F} \beta}$ ist ein Polynom zweiten und $Y_{\mathrm{H} \beta}$ ein Polynom nullten Grades oder vice versa. Dieser Fall ist im Zentralnervensystem wahrscheinlich nicht verwirklicht, weil dann je eine der Leitungen zu den Multiplikatoren trägheitslos wäre.

b) $Y_{\mathrm{F} \beta}$ und $Y_{\mathrm{H} \beta}$ sind beide Polynome ersten Grades; alle Multiplikator-Zuleitungen sind also trägheitsbehaftet. Wir werden daher nur Fall b behandeln. Für $Y_{\mathrm{F} \beta}$ und $Y_{\mathrm{H} \beta}$ ergeben sich also die Gleichungen

bzw.

$$
Y_{\mathrm{F} \beta}=a_{0 \mathrm{~F}}+i k w
$$

$$
Y_{\mathrm{H} \beta}=a_{0 \mathrm{H}}+i k w .
$$

Zählervergleich zwischen Gl. (26 B) und dem zweiten Teil von Gl. (25 D) ergibt

-2 Imaginärteil von $\left(Y_{\mathrm{F} \alpha} Y_{\mathrm{F} \beta}^{*} Y_{\mathrm{H} \alpha}^{*} Y_{\mathrm{H} \beta}\right)=B_{2} k w$.

Die Polynome $Y_{\mathrm{F} \beta}^{*}, Y_{\mathrm{H} \beta}$ wurden schon durch Nennervergleich festgelegt. Also ist das Produkt aus beiden

$Y^{*}{ }_{\mathrm{F} \beta} Y_{\mathrm{H} \beta}=a_{0 \mathrm{~F}} a_{0 \mathrm{H}}+k^{2} w^{2}-\left(a_{0 \mathrm{H}}-a_{0 \mathrm{~F}}\right) i k w$.

In vorstehender Gleichung ist der Imaginärteil von erster Ordnung in $i k w$. Die Polynome $Y_{\mathrm{F} a}$ und $Y^{*} \mathrm{H} \alpha$ können auf Grund der Stabilitätsbedingung höchstens vom ersten Grade in $k w$ sein. Ihre allgemeine Form ist also

bzw.

$$
Y_{\mathrm{F} \alpha}=b_{0 \mathrm{~F}}+b_{1 \mathrm{~F}} i k w
$$

$$
Y_{\mathrm{H} \alpha}=b_{0 \mathrm{H}}+b_{1 \mathrm{H}} i k w .
$$

Setzt man die Gln. (29) und (30) in die Bestimmungsgl. (28) ein, so ist diese nur erfüllt, wenn die Konstanten $b_{1 \mathrm{~F}}$ und $b_{1 \mathrm{H}}$ Null sind. Es ist also

$$
Y_{\mathrm{F} a}=b_{0 \mathrm{~F}} ; \quad Y^{*} \mathrm{H} \alpha=Y_{\mathrm{H} \alpha}=b_{0 \mathrm{H}}
$$

und für den Proportionalitäts-Faktor $B_{2}$ ergibt sich aus den Gln. (28), (29) und (31)

$$
B_{2}=2 b_{0 \mathrm{~F}} b_{0 \mathrm{H}}\left(a_{0 \mathrm{H}}-a_{0 \mathrm{~F}}\right) \text {. }
$$

In dem hier diskutierten Fall $\lambda=4 \Delta s$ bzw. $k=\pi / 2 \Delta s$ reagiert der Käfer in Richtung des zeitlichen Reizablaufes, $R$ liefert also eine zutreffende Information über die Bewegung des Helligkeitsmusters. Daher muß die Konstante $B$ in Gl. (26 A) bzw. $B_{2}$ in Gl. (32) positiv sein. Da wegen $a_{0 \mathrm{H}}>a_{0 \mathrm{~F}}$ der Klammerausdruck in Gl. (32) positiv ist, müssen wir verlangen, daß die Konstanten $b_{0 \mathrm{~F}}$, $b_{0 \mathrm{H}}$ beide positiv oder beide negativ sind. Welcher Fall verwirklicht ist, kann nicht entschieden werden. Eine quantitative Bestimmung von $b_{0 \mathrm{~F}}$ und $b_{0 \mathrm{H}}$ ist nicht möglich, da die auf theoretischem Wege ermittelte Reaktionskurve mit der experimentell bestimmten nur bis auf einen Proportionalitäts-Faktor übereinstimmen kann.

Damit sind die Übertragungs-Funktionen der Filter $\mathrm{F}$ und $\mathrm{H}$ bestimmt. Es ist

und

$$
\begin{gathered}
Y_{\mathrm{F}}= \pm b_{0 \mathrm{~F}} /\left(a_{0 \mathrm{~F}}+p\right) \\
p=i \omega \\
Y_{\mathrm{H}}= \pm b_{0 \mathrm{H}} /\left(a_{0 \mathrm{H}}+p\right) .
\end{gathered}
$$

Durch F ourier-Rücktransformation erhält man aus ihnen die zugeordneten Gewichtsfunktionen

$$
\begin{aligned}
& W_{\mathrm{F}}= \pm b_{0 \mathrm{~F}} e^{-a_{0 \mathrm{~F}} t}, \\
& W_{\mathrm{H}}= \pm b_{0 \mathrm{H}} e^{-a_{0 \mathrm{H}} t} .
\end{aligned}
$$

Sie entsprechen qualitativ den Ergebnissen, die durch Auswertung der Reaktion auf die Bewegung einer Helligkeits-Rauschfunktion gewonnen wurden. Für die Zeitkonstanten $\tau_{\mathrm{F}}, \tau_{\mathrm{H}}$ ergeben sich hier die von den früher angegebenen Werten etwas abweichenden Resultate

$\tau_{\mathrm{F}}=\frac{1}{a_{0 \mathrm{~F}}}=1,6 \pm 0,4$ sec. $\quad \tau_{\mathrm{H}}=\frac{1}{a_{0 \mathrm{H}}}=0,03 \pm 0,02$ sec.

Nachdem die Übertragungs-Eigenschaften der Filter $\mathrm{F}$ und $\mathrm{H}$ festliegen, haben wir noch die der D-Filter zu bestimmen und dazu den zweiten Reaktionsteil [Gl. (26 D)] zu betrachten. Ein Vergleich von Gl. (26 B) mit Gl. (25 D) führt zu der Beziehung

$$
\left|Y_{\mathrm{D}}\right|^{2}=B_{1}|k w| \text {. }
$$

Die Übertragungsfunktion $Y_{\mathrm{D}}$ der D-Filter ist daher

$$
Y_{\mathrm{D}}= \pm \sqrt{B_{1}|p|} \quad \text { mit } p=i \omega .
$$

$Y_{\mathrm{D}}$ wird also durch einen irrationalen Ausdruck in $p$ beschrieben. Nun haben wir gezeigt, daß die allgemeine Form einer Übertragungsfunktion, die aus einer gewöhnlichen Differentialgleichung abgeleitet wird, ein gebrochen rationaler Ausdruck in $p$ sein muß [Gl. (27)]. Daraus wurde geschlossen, daß die D-Filter nicht auf der Basis gewöhnlicher Differentialgleichungen $\mathrm{zu}$ beschreiben sind. Wie schon erwähnt, liegt eine mögliche Alternative in einem Filter mit „verteilten Parametern", das durch eine lineare partielle Differentialgleichung beschrieben wird. Wir werden Einzelheiten, wie physikalische Analogien, Struktur der Differentialgleichung und Stabilität der D-Filter in der Diskussion besprechen. 
Im Anschluß an Gl. (19) haben wir erwähnt, daß die Erscheinungen der „geometrischen Interferenz" auf der Basis dieser Gleichung nur insofern richtig wiedergegeben werden, als sie sich auf Experimente beziehen, in denen der zweite Anteil von Gl. (19 B) konstant gehalten wird. Die Struktur dieses zweiten Anteiles wird von den Übertragungs-Eigenschaften der Filter bestimmt. Legt man die in diesem Abschnitt ermittelten Übertragungsfunktionen zugrunde, so werden auch die Experimente richtig wiedergegeben, in denen die Geschwindigkeits- und Wellenlängen-Abhängigkeit der Reaktionsgleichung eine Rolle spielt ${ }^{7}$. Dies gilt nicht nur für eine, sondern auch für mehrere FourierKomponenten und beweist erneut die Linearität der Filter. Auf Grund dieser Ergebnisse ist zu erwarten, daß man die Reaktion auf eine bewegte Helligkeits-Rauschfunktion durch F o u ri e r - Synthese im Frequenzbereich berechnen kann. Dies soll im folgenden Abschnitt durchgeführt werden.

\section{C. $\beta$. Helligkeitsverteilung: „Weißes“ Rauschen (Synthese im Frequenzbereich)}

Die Analyse der Filter-Übertragungseigenschaften hat zu folgenden Ergebnissen geführt. 1. Die früher angegebene Kinetik der F- und H-Filter wurde bestätigt. Die Werte der Zeitkonstanten $\tau_{\mathrm{F}}, \tau_{\mathrm{H}}$ weichen von den alten Daten ab, liegen jedoch innerhalb derselben Größenordnung. 2. Für den Frequenzgang der D-Filter-Übertragungsfunktion erhielten wir einen Verlauf [Gl. (36 B)], der nicht - wie ursprünglich angenommen - mit $|p|$ (Differentiator, sondern mit $V \mid \overline{p \mid}$ ansteigt.

Es ist das Ziel dieser Untersuchung, eine Theorie aufzustellen, die sowohl die Reaktion auf bewegte sinusförmige Helligkeits-Verteilungen als auch die auf bewegte Helligkeits-Rauschfunktionen richtig wiedergibt. Wir haben daher zu zeigen, daß sich die $1958{ }^{4}$ gemessene Reaktion auf eine bewegte Helligkeits-Rauschfunktion auch aus Gl. (23) errechnen läßt, wenn man die im letzten Abschnitt mit Hilfe des sinusförmigen Helligkeitsmusters bestimmten Übertragungsfunktionen $Y_{\mathrm{F}}, Y_{\mathrm{H}}$ und $Y_{\mathrm{D}}$ heranzieht.

Wir haben schon darauf hingewiesen, daß die AKF von $G(t)$, also der Wechselkomponente der Helligkeits-Rauschfunktion proportional zu einer D i r a c - Funktion ist. Entsprechend muß die Spektraldichte $S(\omega)$ über den ganzen Frequenzbereich konstant sein. Wir setzen $S(\omega)=1$, weil die Reaktion prinzipiell nur bis auf eine ProportionalitätsKonstante bestimmt werden kann. Unter dieser Voraussetzung erhält man für $R$ aus Gl. (23)

$$
R(\Delta t)=\int_{-\infty}^{+\frac{1}{2}}\left|Y_{\mathrm{D}}\right|^{2}\left|\begin{array}{ll}
Y_{\mathrm{F}} & Y^{*} \mathrm{~F} \\
Y_{\mathrm{H}} & Y^{*}{ }_{\mathrm{H}}
\end{array}\right| e^{+i \omega \Delta t} \mathrm{~d} \omega
$$

Nun ist aber $\left|Y_{\mathrm{D}}\right|^{2}\left|\begin{array}{cc}Y_{\mathrm{F}} & Y^{*} \mathrm{~F} \\ Y_{\mathrm{H}} & Y^{*}{ }_{\mathrm{H}}\end{array}\right|$ eine antisymmetrische Funktion, so daß sich Gl. (37 A) reduziert auf

$R(\Delta t)=\int_{0}^{+\infty}\left|Y_{\mathrm{D}}\right|^{2}\left|\begin{array}{cc}Y_{\mathrm{F}} & Y^{*} \mathrm{~F} \\ Y_{\mathrm{H}} & Y^{*}{ }_{\mathrm{H}}\end{array}\right| \cdot i \sin \omega \Delta t \mathrm{~d} \omega$.

Trägt man in vorstehender Gleichung die Übertragungsfunktionen $Y_{\mathrm{F}}, Y_{\mathrm{H}}$ und $Y_{\mathrm{D}}$ ein und setzt $\alpha=\tau_{\mathrm{F}} / \tau_{\mathrm{H}} ; z=w \tau_{\mathrm{F}} / \Delta s$, so ergibt sich mit dem Normierungsfaktor $N$ als Lösung des Integrals

$$
\begin{array}{r}
R(z)=N\left[\frac{\alpha}{z} e^{-\alpha / z} \overline{E i}\left(\frac{\alpha}{z}\right)-\frac{\alpha}{z} e^{+\alpha / z} E i\left(-\frac{\alpha}{z}\right)\right. \\
\left.-\frac{1}{z} e^{-1 / z} E i\left(\frac{1}{z}\right)+\frac{1}{z} e^{+1 / z} E i\left(-\frac{1}{z}\right)\right]
\end{array}
$$

$E i(-x)$ und $\bar{E} i(x)$ mit $x=\alpha / z$ sind wie folgt definiert

$$
\begin{array}{rlrl}
-E i(-x) & =\int_{-\infty}^{-x} \frac{e^{t}}{-t} \mathrm{~d} t & & 0<x<\infty \\
\overline{E i}(x) & =l i e^{x} \quad \text { und } & l i x=\int_{0}^{x} \frac{\mathrm{d} t}{\ln t} .
\end{array}
$$

Beide Funktionen sind tabelliert und können Tabellenwerken entnommen werden ${ }^{15}$. Setzt man die Werte der Zeitkonstanten $\tau_{\mathrm{F}}$ und $\tau_{\mathrm{H}}$ aus Gl. (35) in Gl. (38) ein und wählt als Basis $\Delta s=14^{\circ}$, so ergibt sich der in Abb. 4 dargestellte Reaktionsverlauf

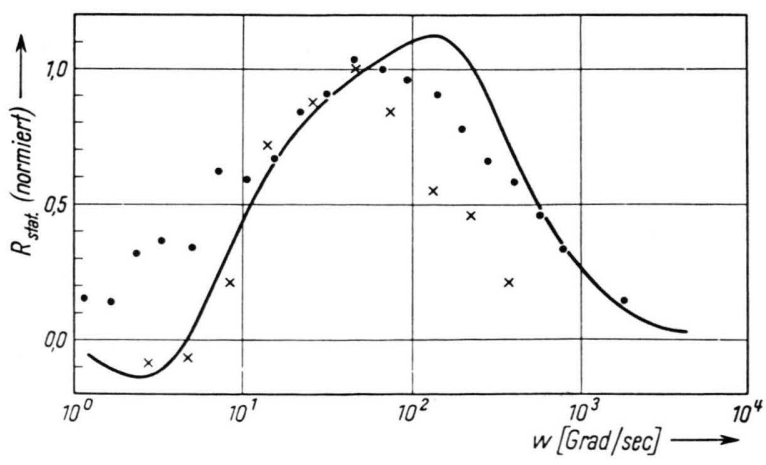

Abb. 4. Die Reaktionsgröße der verallgemeinerten Funktionsstruktur als Funktion der Geschwindigkeit einer „weißen“ Helligkeits-Rauschfunktion (ausgezogene Kurve). Basis $\Delta s=14^{\circ}$. Meßpunkte aus zwei verschiedenen Experimenten mit statistischen Helligkeitsverläufen. 1. Punkte: Zylinder aus $7^{\circ}$ breiten Streifen. 2. Kreuze: Zylinder aus $1^{\circ}$ breiten Streifen. Aufeinanderfolge der Streifen in beiden Fällen statistisch.

15 Siehe z. B. Jahnke u. Emde: Tafeln höherer Funktionen. Teubner Verlagsgesellschaft, Leipzig 1948. 
(ausgezogene Kurve). Bemerkenswert an der theoretischen Reaktionskurve ist die negative Phase bei kleinen Geschwindigkeitswerten. Auf Grund der Theorie wird also in diesem Geschwindigkeitsbereich eine optomotorische Reaktion erwartet, die der Bewegung der Helligkeits-Rauschfunktionen entgegengerichtet ist. Dabei muß man sich nochmals vergegenwärtigen, daß die Theorie von zwei Annahmen Gebrauch macht, die experimentell nicht streng zu verwirklichen sind. 1. Verschwindend kleiner Apertur der Ommatidien. 2. Eine dem Grenzfall des „weiBen" Rauschens entsprechende Helligkeits-Verteilung.

Abb. 4 enthält zwei Reaktionskurven, die unter verschiedenen experimentellen Bedingungen aufgenommen wurden ${ }^{5}$. 1. Punkte: Die HelligkeitsRauschfunktion bestand aus $7^{\circ}$ breiten Streifen mit unterschiedlichen Helligkeitswerten, die auf einem Zylinder in statistischer Weise aufgebracht waren. Zwischen Käferauge und bewegtem statistischem $\mathrm{Zy}$ linder befand sich ein feststehender Zylinder mit $1^{\circ}$ breiten und $14^{\circ}$ voneinander entfernten senkrechten Schlitzen. Der Abb. 4 ist zu entnehmen, daß die ausgemessene Reaktion sich zwar angenähert im vorausgesagten Geschwindigkeitsbereich befindet, jedoch quantitativ von der berechneten Reaktionskurve abweicht. Diese Abweichung ist besonders auffällig im Bereich kleiner Geschwindigkeiten, da keine - der Bewegungsrichtung des Zylinders entgegengerichtete - Reaktion beobachtet wurde. 2. Kreuze: In diesem Fall bestand die Helligkeits-Rauschfunktion aus $1^{\circ}$ breiten Streifen. Eine optomotorische Reaktion konnte aber nur dann ausgemessen werden, wenn das freie Insektenauge (ohne Schlitzzylinder) die Bewegung rezipierte. Unter diesen Bedingungen werden vom Zentralnervensystem nicht nur Reize über die Abtastbasis $\Delta s=14^{\circ}$, sondern auch über die Basis $\Delta s=7^{\circ}$ (Nachbarommatidien) ausgewertet. Für die Theorie bedeutet dies, daß der Reaktionsverlauf sich additiv aus der in Abb. 4 aufgetragenen Kurve und derselben, allerdings um den Faktor zwei nach links verschobenen, Reaktionskurve zusammensetzt. Wir haben diesen zweiten theoretischen Reaktionsverlauf in Abb. 4 nicht eingetragen, da er sich abgesehen von einer Verschiebung um den Faktor $\sqrt{2}$ zu kleineren Geschwindigkeitswerten nicht wesentlich von der in Abb. 4 enthaltenen Reaktionskurve unterscheidet. Aus Abb. 4 ist zu ersehen, daß die Meßpunkte im ansteigenden Reaktionsteil die wesentliche Forderung der Theorie erfüllen: Bei langsamen Zylinder-Geschwindigkeiten tritt eine Re- aktion auf, die der Bewegung der Helligkeits-Rauschfunktion entgegengerichtet ist. Wesentliche Abweichungen von der theoretischen Reaktionskurve treten erst bei Zylinder-Geschwindigkeiten $w>60^{\circ} / \mathrm{sec}$ auf. Die folgende Überlegung liefert eine Vorstellung von den Ursachen für diese Abweichungen: Der theoretisch ermittelte Kurvenverlauf in Abb. 4 entstand aus einer Superposition von Teilreaktionen auf die F o u rier-Komponenten einer „weißen“ Helligkeits-Rauschfunktion [Gl. (37)]. Definitionsgemäß treten diese Komponenten im Wellenlängenbereich $0 \leqq \lambda<+\infty$ mit konstanter Amplitude auf. Eine Analyse der Reaktionen auf die Bewegung sinusförmiger Helligkeitsmuster [Gl. (26 A)] hat gezeigt ${ }^{7}$, daß Muster großer Wellenlänge $(\lambda \gg 4 \Delta s)$ zu Reaktionskurven führen, die im Bereich hoher ZylinderGeschwindigkeiten liegen. Verkleinert man die Wellenlänge des Helligkeitsmusters, so verschieben sich die Reaktionskurven zu kleineren Geschwindigkeiten. Bestimmt man die resultierende Reaktion auf eine bewegte Helligkeitsverteilung, die sich aus einem Wellenlängen-Kontinuum $(0 \leqq \lambda<+\infty)$ von $\mathrm{F}$ o u ri e r-Komponenten zusammensetzt, so wird die Reaktion - wegen der Übertragungs-Eigenschaften der D-, F- und H-Filter - bei großen Geschwindigkeiten im wesentlichen durch den langwelligen, bei kleinen Geschwindigkeiten des Musters durch den kurzwelligen Teil des Kontinuums bestimmt. Die Annäherung einer „weißen“ Helligkeits-Rauschfunktion durch Zylinder mit statistisch aufgebrachten Helligkeitsstreifen hat zur Folge: 1. Die verwirklichte Rauschfunktion setzt sich nicht aus einem Kontinuum, sondern einem Linienspektrum zusammen, da das Muster ein Periodizitäts-Intervall $\left(360^{\circ}\right)$ besitzt. Spektrallinien von Wellenlängen $\lambda>360^{\circ}$ treten in der Verteilung nicht auf. 2. Die Amplituden des Linienspektrums nehmen mit fallender Wellenlänge ab. Der Abfall erfolgt bei um so kleineren Wellenlängen, je geringer die Streifenbreite der einzelnen Helligkeitswerte gewählt wird. Während bei breiten Streifen die Spektraldichte der Verteilung ihre wesentlichen Anteile bei großen Wellenlängen (jedoch $\lambda<360^{\circ}$ ) besitzt, liegen sie bei sehr schmalen Streifen im Bereich kleiner Wellenlängen. Aus diesen Gründen ist zu erwarten, daß die gemessenen Reaktionen auf die Bewegung eines Zylinders aus $1^{\circ}$ breiten Streifen im Bereich kleiner Geschwindigkeiten mit der berechneten Reaktionskurve übereinstimmen. Das Gegenteil gilt für den Zylinder aus $7^{\circ}$ breiten Streifen. Hier sollte und ist die Übereinstim- 
mung zwischen Theorie und Experiment bei größeren Zylinder-Geschwindigkeiten besser, da die langwelligen Komponenten im Spektrum überwiegen.

Zum Abschluß dieses Kapitels kommen wir noch einmal auf die mit einem statistischen $1^{\circ}$-Streifenzylinder verifizierte Gegenreaktion zurück, die bei kleinen Mustergeschwindigkeiten auftritt. Aus der Theorie folgt dieses Ergebnis formal durch Integration über die konstante Spektraldichte der „weiBen" Helligkeits-Rauschfunktion. Anschaulich bedeutet es, daß in diesem Fall von einem der Korrelatoren in der Funktionsstruktur bevorzugt (also mehr als 50\%) plus-minus Signalkombinationen verrechnet werden. Die transformierten Größen $L^{*}{ }_{\mathrm{AF}}$, $L^{*}{ }_{\mathrm{AH}}, L^{*}{ }_{\mathrm{BF}}, L^{*}{ }_{\mathrm{BH}}$ müssen also sowohl positive als auch negative Funktionsanteile besitzen. Das dies der Fall sein muß, folgt aus der Tatsache, daß der Mittelwert der Helligkeitsverteilung schon von den D-Filtern [s. Gl. (36 B) ] und nicht erst von der Subtraktionsinstanz entfernt wird.

\section{Diskussion der Ergebnisse}

Die vorliegende Theorie des Bewegungssehens von Chlorophanus geht von einem linearen Ansatz für die beteiligten zentralnervösen Übertragungselemente (Filter) aus. Dieser Ansatz wird durch Experimente nahegelegt, in denen die linearen ÜbertragungsEigenschaften der Filter im Einzelversuch ${ }^{3,5}$ bestimmt wurden. Er hat zur Folge, daß sich die berechnete Reaktion auf sinusförmige Helligkeits-Verteilungen aus zwei Anteilen multiplikativ zusammensetzt, wobei der eine von der Basis $\Delta s$ und der Wellenlänge des sinusförmigen Musters, der andere von der Wellenlänge, der Geschwindigkeit des Musters und den Zeitkonstanten der Filter abhängt. Eine weitere Konsequenz des linearen Ansatzes ist, daß die Reaktion auf eine bewegte HelligkeitsRauschfunktion aus der Reaktionsgleichung für eine bewegte sinusförmige Helligkeits-Verteilung durch Integration (Aufsummieren) über alle in der Verteilung vorkommende Partialfrequenzen berechenbar sein muß. Das Ergebnis dieser Synthese der Reaktion führte zur Übereinstimmung mit den experimentellen Befunden.

Auf Grund dieser Übereinstimmung zwischen Theorie und Experiment schließen wir: Die Nachrichtenübertragung von den Rezeptoren bis zu den zentralnervösen Korrelations-Instanzen ist eine lineare; es gilt das Superpositions-Prinzip.
Für die Verwirklichung derartiger ÜbertragungsEigenschaften ergeben sich prinzipiell drei Denkmöglichkeiten :

1. Die im Übertragungskanal Rezeptor-Korrelator auftretenden Nachrichten-Transformationen sind linear. Unter diesen Umständen ist auch die Übertragungs-Eigenschaft des Kanales linear. Die Transformationen sind kommutierbar; ihre Reihenfolge im Übertragungskanal ist beliebig.

2. Die im Übertragungskanal auftretenden Nachrichten-Transformationen sind zum Teil nichtlinear. Jede nichtlineare Transformation wird jedoch durch die zu ihr inverse rückgängig gemacht. Die Reihenfolge der Transformationen im Kanal ist nicht beliebig.

3. Die auftretenden Transformationen sind z. T. nichtlinear. Sie werden aber durch einen Gegenkoppelungszweig zwischen Rezeptor und Korrelator linearisiert.

Eine Entscheidung darüber, welche dieser drei Realisierungs-Möglichkeiten im Zentralnervensystem des Käfers verwirklicht ist, kann nur im direkten Zugriff gefällt werden.

Aus den allgemeinen Reaktionsgl. (9) und (23) läßt sich entnehmen, daß Richtung und Stärke der optomotorischen Wendereaktion von der AKF bzw. Spektraldichte der bewegten Helligkeits-Verteilung abhängt. Darin liegt eine Besonderheit: Sowohl die AKF als auch die Spektraldichte einer Helligkeitsverteilung enthalten nur $50 \%$ der in einem Helligkeitsmuster vorhandenen Information. Welcher Informationsanteil in der Funktionsstruktur verlorengeht, läßt sich aus der Definitionsgleichung für die Spektraldichte $S(\omega)$ ablesen ${ }^{9}$.

Es ist

$$
S(\omega)=\lim _{T \rightarrow \infty} \frac{c_{T}(\omega) c_{T}^{*}(\omega)}{T}
$$

mit $\quad c_{T}(\omega)=\frac{1}{\sqrt{2 \pi}} \int_{-T}^{+T} G(t) e^{-i \omega t} \mathrm{~d} t$.

In diesem Fall bedeuten $c_{T}$ die komplexe Spektralfunktion von $G(t)$ und $T$ ein Zeitintervall. Da $S(\omega)$ durch das Produkt aus $c_{T}$ und dem konjugiert komplexen $c^{*}{ }_{T}$ gebildet wird, ist die Spektraldichte unabhängig von den Phasenlagen der in $G(t)$ enthaltenen F o u r i e r - Komponenten. Läßt sich die Wechselkomponente eines periodischen Helligkeitsmusters z. B. durch $N$ F o u ri e r - Komponenten vollständig darstellen, so ist das Muster durch $2 N$ Daten bestimmt. $N$ dieser Daten charakterisieren die Amplitu- 
den, $N$ Daten die Phasenlagen der einzelnen Komponenten. Denkt man sich also ein bewegtes Helligkeitsmuster aus F o u r i e r - Komponenten aufgebaut, deren Amplituden und Phasen durch die Struktur des Musters bestimmt sind, so läßt sich zusammenfassend sagen:

1. Die in den Phasenlagen enthaltene Information über die Struktur der optischen Umwelt geht in der Funktionsstruktur für das Bewegungssehen verloren und hat daher keinen Einfluß auf die Reaktion.

2. Da die Phasen der F o u ri e r-Komponenten $50 \%$ der in einer Helligkeits-Verteilung enthaltenen Information tragen, wird die Reaktion $R$ nur von den restlichen 50\%, die in den Amplituden der einzelnen Komponenten enthalten sind, beeinflußt.

3. Die optomotorische Reaktion von Chlorophanus muß also invariant gegenüber allen Transformationen sein, die nur auf die Phasen der - in einer Helligkeits-Verteilung enthaltenen - FourierKomponenten einwirken. Damit ist jeweils eine Klasse von für das menschliche Auge verschiedenen Helligkeits-Verteilungen (Abb. 5 enthält hierfür ein Beispiel) ausgezeichnet, die von dem optomotorischen Auswertesystem des Käfers nicht unterschieden werden sollten; diese Konsequenz ist experimentell nachgeprüft und bestätigt worden ${ }^{5,7}$.

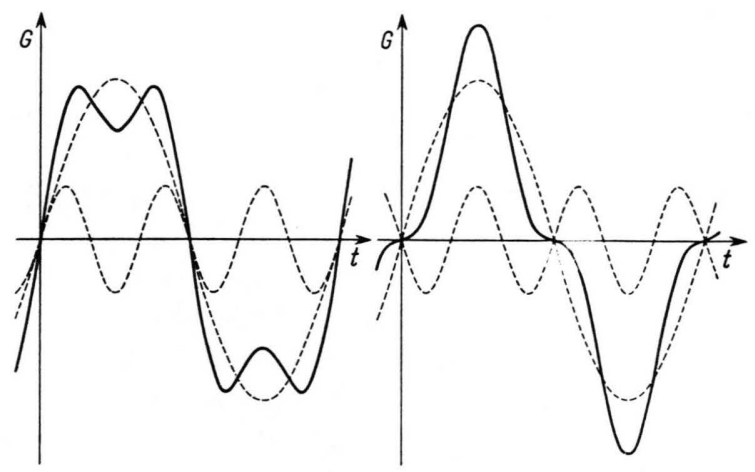

Abb. 5. Zwei verschiedene Helligkeitsmuster (ausgezogene Kurven), die durch Ubberlagerung zweier $\mathrm{F}$ o u ri e $\mathrm{r}$ - Komponenten mit verschiedener gegenseitiger Phasenlage hergestellt wurden. Näheres s. Text.

Die Analyse der Reaktionskurven hat auf zwei Typen von zentralnervösen Filtern geführt. Die Filter $\mathrm{F}$ und $\mathrm{H}$ gehören dem einen, die Filter $\mathrm{D}$ dem anderen Typ an. Sie unterscheiden sich in ihren Übertragungsfunktionen. Die Übertragungsfunktionen der Filter $\mathrm{F}$ und $\mathrm{H}$ sind rationale, die der Filter $\mathrm{D}$ irrationale Funktionen der Frequenz $\omega$. Wie wir im Zusammenhang mit den Gln. (26) bis (31) gezeigt haben, folgt daraus, daß nur die Filter $\mathrm{F}$ und $\mathrm{H}$ durch gewöhnliche Differentialgleichungen mit konstanten Koeffizienten zu beschreiben sind. Die speziellen Übertragungsfunktionen von $\mathrm{F}$ und $\mathrm{H}$ entsprechen einer Reaktionskinetik erster Ordnung und damit auch einer gewöhnlichen Differentialgleichung erster Ordnung. Da die Lösung dieser Gleichung eine Anfangsbedingung enthält, ist das Filter in jedem Zeitpunkt durch Angabe eines Parameters vollständig beschrieben. Es besitzt eine Koordinate bzw. einen Freiheitsgrad. Ein physikalisches Modell, das dieser Kinetik entspricht, enthält einen unabhängigen Energiespeicher. Das allgemeine Kennzeichen von Filtern, deren Übertragungs-Eigenschaften auf gewöhnliche Differentialgleichungen $n$-ter Ordnung führen, besteht darin, daß sie durch $n$ Koordinaten - also eine endliche Anzahl - eindeutig beschrieben werden können.

Die Übertragungsfunktion der D-Filter läßt sich nicht aus einer gewöhnlichen Differentialgleichung ableiten, da sie ein irrationaler Ausdruck in $\omega$ ist. Eine mögliche Alternative liegt in einer partiellen Differentialgleichung. Im Gegensatz zu den Filtern, die durch eine endliche Anzahl von Koordinaten zu beschreiben sind, ist der jeweilige Zustand eines Filters, dessen Übertragungs-Eigenschaften auf eine partielle Differentialgleichung führen, nur durch die unendliche Mannigfaltigkeit der Werte einer oder mehrerer voneinander unabhängiger Raumfunktionen eindeutig festgelegt. Ein eindimensionales physikalisches Beispiel hierfür ist eine elektrische Leitung, deren jeweiliger Zustand durch die Angabe zweier Funktionen - der räumlichen Verteilung von Strom und Spannung auf der Leitung - vollständig charakteristiert ist.

Wir haben untersucht, welcher partiellen Differentialgleichung die Übertragungsfunktion des D-Filters [Gl. (36 B) ] entspricht. Es zeigte sich, daß eine spezielle Lösung der Gleichung

$$
\beta \frac{\partial^{2}}{\partial x^{2}} L^{*}(x, t)=\frac{\partial}{\partial t} L^{*}(x, t)
$$

im Frequenzbereich die geforderten Übertragungs-Eigenschaften besitzt. $L^{*}$ in Gl. (41) ist im Hinblick auf die Funktionsstruktur die zeit-- und ortsabhängige Variable des Filterausganges, $x$ eine Ortskoordinate und $\beta$ eine dimensionsbehaftete Konstante. Durch Gl. (41) werden verschiedene eindimensionale physikalische Phänomene beschrieben; so z. B. die Ausbreitung einer elektrischen Spannung längs einer induktionslosen und ableitungs- 
freien Leitung (sogenanntes Th o m s o n-Kabel)*, die Wärmeleitung in einem Medium mit der Temperaturleitzahl $\beta$ und die Diffusion eines Gases oder z. B. von Elektronen in einem Leiter.

Die Übertragungsfunktion des D-Filters leiten wir aus der Analogie eines rückwirkungsfreien T h o m s o n Kabels her. Unter diesen Umständen geht Gl. (41) in die Modellgleichung

$$
\frac{1}{r \cdot c} \frac{\partial^{2}}{\partial x^{2}} u(x, t)=\frac{\partial}{\partial t} u(x, t)
$$

über. Hierin ist $u(x, t)$ die Spannung an der Stelle $x$ zur Zeit $t, r$ der Kabelwiderstand und $c$ die Querkapazität pro Längeneinheit. Gl. (41 A) läßt sich in die beiden gekoppelten Gleichungen

$$
\begin{aligned}
& -\frac{\partial}{\partial x} u(x, t)=r \cdot i(x, t), \\
& -\frac{\partial}{\partial x} i(x, t)=c \frac{\partial}{\partial t} u(x, t)
\end{aligned}
$$

zerlegen, in denen $i(x, t)$ der Kabelstrom an der Stelle $x$ zur Zeit $t$ bedeutet. Transformiert man Gl. (42) in den Unterbereich der Laplace (L)-Transformation und bezeichnet mit $U(x, p)$ die L-Transformierte von $u(x, t)$ mit $I(x, p)$ die L-Transformierte von $i(x, t)$ und mit $p$ die komplexe Unterbereichsvariable, so ergeben sich die Gleichungen

$$
\begin{aligned}
& -\frac{\partial}{\partial x} U(x, p)=r I(x, p) \\
& -\frac{\partial}{\partial x} I(x, p)=c p U(x, p)-c u(x, 0)
\end{aligned}
$$

worin $u(x, 0)$ die Spannungsverteilung über das Kabel zur Zeit $t=0$ ist. Wir setzen nun folgende Anfangs- und Randbedingungen des Problemes fest:

1. Für Zeiten $t<0$ sei die Spannung an jeder Stelle des Kabels null, also $u(x, t)=0$ für $t<0$.

2. Im Zeitpunkt $t=0$ wird ein delta-funktionsförmiger Spannungsstoß an den Kabelanfang $(x=0)$ gelegt.

3. Das betrachtete Th o m s on-Kabel sei rückwirkungsfrei; eine Störung, die vom Kabelanfang ausgeht, wird vom Kabelende nicht reflektiert. Um dies zu erreichen, ist in der Theorie anzunehmen, daß das Kabel unendlich ausgedehnt ist. Praktisch läßt sich dieser Fall mit endlicher Kabellänge erreichen, wenn man das Kabelende mit seinem Wellenwiderstand abschließt. Man erhält unter diesen Bedingungen aus Gl. (43) die Übertragungsfunktionen des Kabels ${ }^{16}$

$$
\begin{gathered}
U(x, p)=e^{-\sqrt{r c p} x} \\
I(x, p)=\frac{1}{\sqrt{(r / c)}} \sqrt{p} e^{-\sqrt{r c p} x}
\end{gathered}
$$

und durch Rücktransformation in den Zeitbereich die zugeordneten Gewichtsfunktionen für Spannung und Strom.

* Ein Th o m s o n-Kabel ist eine elektrische Leitung, in der nur Längswiderstände und Querkapazitäten pro Längeneinheit (verteilte Parameter) auftreten.

$$
\begin{array}{r}
v(x, t)_{\delta}=\frac{x \sqrt{r \cdot c}}{2 \sqrt{\pi} \sqrt{t^{3}}} e^{-x^{2} r c / 4 t}, \\
i(x, t)_{\delta}=\frac{r c x^{2}-2 t}{4 \sqrt{\pi} \sqrt{(r / c)} V^{5}} e^{-x^{2} r c / 4 t} .
\end{array}
$$

Ein Vergleich zwischen der Übertragungsfunktion des Kabels [Gl. (44)] und der des D-Filters [Gl. (36 B) ] zeigt, daß $I(x, p)$ denselben AmplitudenFrequenzgang besitzt wie $Y_{\mathrm{D}}(p)$, wenn man den Strom am Kabeleingang betrachtet $(x=0)$. Diese Analogie erstreckt sich jedoch nur auf den Amplitudengang von $Y_{\mathrm{D}}$, da der Phasengang der D-Filter keinen Einfluß auf die Reaktion besitzt und daher aus der Systemanalyse auch nicht zu erschließen ist.

Bevor wir diese Analogie näher betrachten, ist es notwendig, die Stabilität der D-Filter zu untersuchen. Innerhalb der Funktionsstruktur, also in Verbindung mit den Filtern $\mathrm{F}$ und $\mathrm{H}$, verhalten sich die D-Filter stabil. Experimentell folgt dies aus der Messung graduierter Wendereaktionen und kommt mathematisch darin zum Ausdruck, daß die Synthese der Reaktion gegenüber bewegten Helligkeits. Rauschfunktionen auf konvergente Integrale in den Gln. (37 A, B) führt. Betrachtet man dagegen die D-Filter isoliert, so sind sie instabil. Das ist unmittelbar einzusehen: Wird die zur Übertragungsfunktion $Y_{\mathrm{D}}$ bzw. $I(0, p)$ gehörende Gewichtsfunktion $i(0, t)_{\delta}$ aus Gl. (45 B) dem Stabilitätskriterium in Gl. (3) unterworfen, so divergiert das Integral

$$
\int_{-\infty}^{+\infty}\left|i(0, t)_{\delta}\right| \mathrm{d} t .
$$

Sind beide Filtertypen nicht nur formal in der Funktionsstruktur, sondern auch räumlich im Zentralnervensystem getrennt, müssen die D-Filter auch für sich stabil sein und eine endliche obere Grenzfrequenz besitzen. Vermutlich wird die Übertragungsfunktion $Y_{\mathrm{D}}$ daher nicht nur durch den in Gl. (36 B) enthaltenen Verlauf, sondern durch Gl. (44 B) für $x>0$ wiedergegeben. Unter diesen Umständen sind die D-Filter auch isoliert stabil, was man durch Einsetzen der entsprechenden Gewichtsfunktionen in das Stabilitätskriterium Gl. (3) beweisen kann. Wir haben untersucht, welchen Einfluß der Faktor $e^{-\sqrt{r c p} x}$ in der Übertragungsfunktion $Y_{\mathrm{D}}$ auf die Reaktionskurve in Abb. 3 besitzt, wenn man $\sqrt{r c x}$

16 Siehe z. B. K. W. Wagner, Operatorenrechnung und L a p l a c e sche Transformation. J.A.Barth, Leipzig 1950; E. Weber, Linear Transient Analysis Vol. II, John Wiley, New York 1956. 
variiert. Es zeigte sich, daß mit wachsendem $V r c x$ die Symmetrie zwischen dem ansteigenden und dem abfallenden Reaktionsschenkel mehr und mehr verlorengeht, was nicht mit den Meßergebnissen übereinstimmt. Praktisch läßt sich jedoch die aufgetragene Reaktionskurve, die dem Wert $\sqrt{r c} x=0$ entspricht, nicht von Kurvenläufen unterscheiden, für die $\sqrt{r c x}$ kleiner als $10^{-2}$ ist. Da $\sqrt{r c} x$ die Dimension $\sqrt{\text { Zeit }}$ besitzt, folgt daraus, daß die Zeitkonstante der D-Filter kleiner als $10^{-4}$ sein muß. Die D-Filter beschreiben möglicherweise die Trans- formation der Lichtenergie in elektrische Potentiale, da die schnellsten synaptischen Übertragungsprozesse in der Größenordnung von $7 \cdot 10^{-4}$ Sek. liegen. Wegen der Rückführbarkeit der Übertragungsfunktion $Y_{\mathrm{D}}$ auf die partielle Differentialgleichung, erscheint es denkbar, daß diese Übersetzung auf einem lichtabhängigen elektronischen Diffusionsprozeß beruht.

Wir danken Herrn Dr. B. Hassenstein für zahlreiche wertvolle Diskussionen.

\section{NOTIZEN}

\section{The Effect of Complex Formation on the Infra-red Spectra of Titanium Tetrafluoride and Tetrachloride}

By G. S. Rao

Department of Chemistry, University of Saugar, India (Z. Naturforschg. 14 b, 689-690 [1959]; eingegangen am 27. April 1959)

Titanium tetrafluoride forms a $1: 2$ complex with pyridine and $1: 1$ complexes with acetonitrile, propionitrile and benzonitrile while titanium tetrachloride forms 1:2 complexes with all these ligands ${ }^{1}$. The

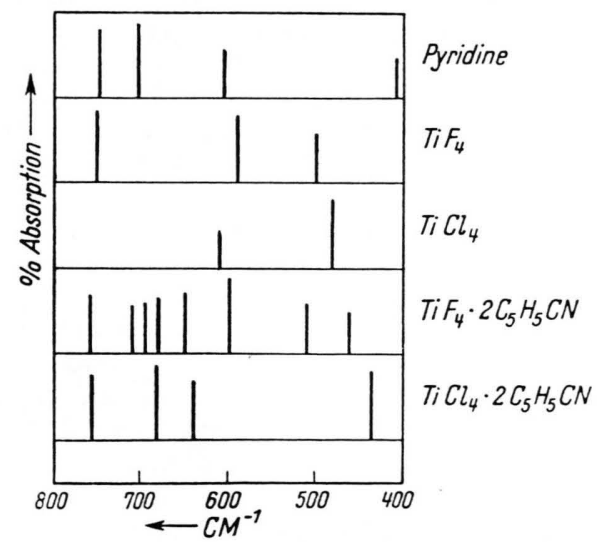

Fig. 1. Infra-red Spectra of Pyridin Complexes.

infra-red spectra of titanium tetrafluoride, tetrachloride, the organic ligands viz. pyridine, acetonitrile, propionitrile and benzonitrile and the complexes referred above have been measured in the region $400-800 \mathrm{~cm}^{-1}$ (Potassium bromide prism) ${ }^{2}$, using a Perkin Elmer Model 21 Spectrophotometer. All the measurements were made on Nujol or hexachlorobutadiene nulls of the complexes and the preparation of nulling was carried out in a dry box. The frequency changes involved in the above spectra are shown graphically in Figs. $1-4$.

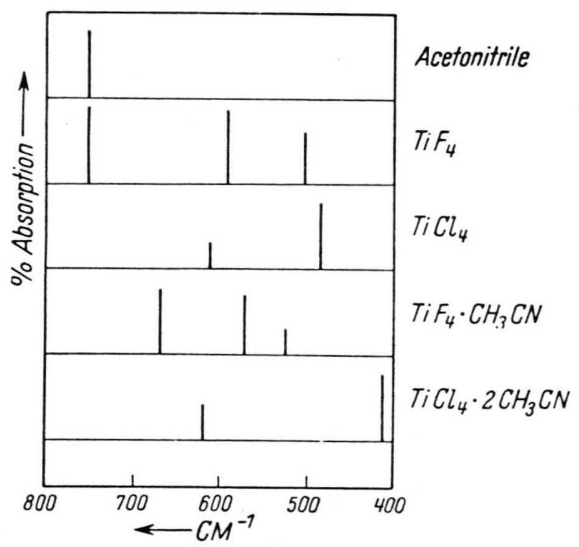

Fig. 2. Infra-red Spectra of Acetonitrile Complexes.

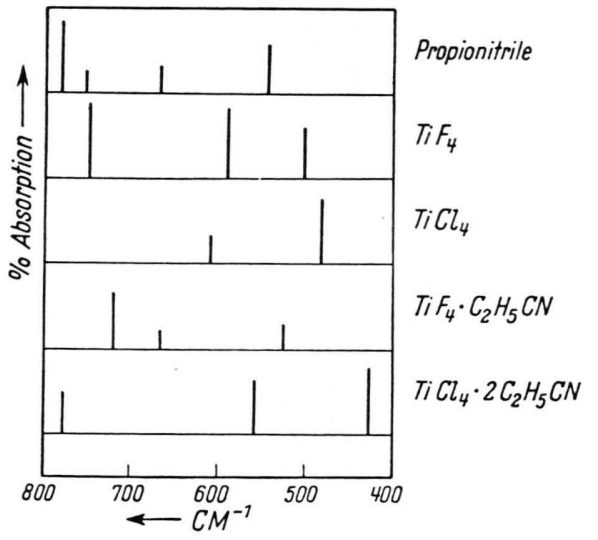

Fig. 3. Infra-red Spectra of Propionitrile Complexes.

In the case of titanium tetrachloride the infra-red active Ti-Cl bond stretching frequency occurs at 482 $\mathrm{cm}^{-1}$ (495 $\mathrm{cm}^{-1}$ in the $\mathrm{R}$ a m a n spectrum) and on complex formation it is usually shifted markedly to low frequencies implying a probable weakening of the $\mathrm{Ti}-\mathrm{Cl}$ 